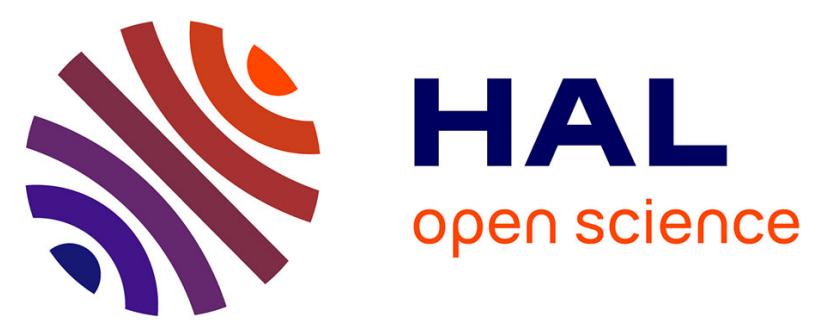

\title{
Morphological processing of univariate Gaussian distribution-valued images based on Poincaré upper-half plane representation
}

Jesus Angulo, Santiago Velasco-Forero

\section{To cite this version:}

Jesus Angulo, Santiago Velasco-Forero. Morphological processing of univariate Gaussian distributionvalued images based on Poincaré upper-half plane representation. Frank Nielsen. Geometric Theory of Information, Springer International Publishing, pp.331-366, 2014, Signals and Communication Technology, 978-3-319-05316-5. 10.1007/978-3-319-05317-2_12 . hal-00795012v3

HAL Id: hal-00795012

https://hal-mines-paristech.archives-ouvertes.fr/hal-00795012v3

Submitted on 22 Jan 2015

HAL is a multi-disciplinary open access archive for the deposit and dissemination of scientific research documents, whether they are published or not. The documents may come from teaching and research institutions in France or abroad, or from public or private research centers.
L'archive ouverte pluridisciplinaire HAL, est destinée au dépôt et à la diffusion de documents scientifiques de niveau recherche, publiés ou non, émanant des établissements d'enseignement et de recherche français ou étrangers, des laboratoires publics ou privés. 


\title{
Morphological processing of univariate Gaussian distribution-valued images based on Poincaré upper-half plane representation
}

\author{
Jesús Angulo $^{a}$, Santiago Velasco-Forero ${ }^{b}$ \\ ${ }^{a}$ CMM-Centre de Morphologie Mathématique, Mathématiques et Systèmes, \\ MINES ParisTech; France \\ ${ }^{b}$ National University of Singapore, Department of Mathematics \\ jesus.angulo@mines-paristech.fr,matsavf@nus.edu.sg
}

January 2014

\begin{abstract}
Mathematical morphology is a nonlinear image processing methodology based on the application of complete lattice theory to spatial structures. Let us consider an image model where at each pixel is given a univariate Gaussian distribution. This model is interesting to represent for each pixel the measured mean intensity as well as the variance (or uncertainty) for such measurement. The aim of this work is to formulate morphological operators for these images by embedding Gaussian distribution pixel values on the Poincaré upper-half plane. More precisely, it is explored how to endow this classical hyperbolic space with various families of partial orderings which lead to a complete lattice structure. Properties of order invariance are explored and application to morphological processing of univariate Gaussian distribution-valued images is illustrated.

Keywords: Ordered Poincaré half-plane, hyperbolic partial ordering, hyperbolic complete lattice, mathematical morphology, Gaussian-distribution valued image, information geometry image filtering
\end{abstract}

\section{Introduction}

This work is motivated by the exploration of a mathematical image model $f$ where, instead of having a scalar intensity $t \in \mathbb{R}$ at each pixel $p$, i.e., $f(p)=t$, we have a univariate Gaussian probability distribution of intensities $N\left(\mu, \sigma^{2}\right) \in \mathcal{N}$, i.e., image $f$ is defined as the function

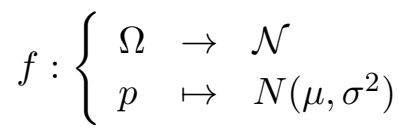


where $\Omega$ is the support space of pixels $p$ (e.g., for $2 \mathrm{D}$ images $\Omega \subset \mathbb{Z}^{2}$ ) and $\mathcal{N}$ denotes the family of univariate Gaussian probability distribution functions (pdf). Nowadays most of imaging sensors only produce single scalar values since the CCD (charge-coupled device) cameras typically integrates the light (arriving photons) during a given exposure time $\tau$. To increase the signal-to-noise ratio (SNR), exposure time is increased to $\tau^{\prime}=\alpha \tau, \alpha>1$. Let suppose that $\alpha$ is a positive integer number, this is equivalent to a multiple acquisition of $\alpha$ frames during $\tau$ for each frame (i.e., a kind of temporal oversampling). The standard approach only considers the sum (or average) of the multiple intensities [28], without taking into account the variance which is a basic estimator of the noise useful for probabilistic image processing. Another example of such a representation from a gray scale image consists in considering that each pixel is described by the mean and the variance of the intensity distribution from its centered neighboring patch. This model has been for instance recently used in [10] for computing local estimators which can be interpreted as pseudo-morphological operators.

Let us consider the example of gray scale image parameterized by the mean and the standard deviation of patches given in Fig. 1. We observe that the underlying geometry of this space of patches is not Euclidean, e.g., the geodesics are clearly curves. In fact, as we discuss in the paper, this parametrization corresponds to one of the models of hyperbolic geometry.

Henceforth, the corresponding image processing operators should be able to deal with Gaussian distributions-valued pixels. In particular, morphological operators for images $f \in$ $\mathcal{F}(\Omega, \mathcal{N})$ involve that the space of Gaussian distributions $\mathcal{N}$ must be endowed of a partial ordering leading to a complete lattice structure. In practice, it means that given a set of Gaussian pdfs, as the example given in Fig. 2, we need to be able to define a Gaussian pdf which corresponds to the infimum (inf) of the set and another one to the supremum (sup). Mathematical morphology is a nonlinear image processing methodology based on the computation of sup/inf-convolution filters (i.e., dilation/erosion operators) in local neighborhoods [31]. Mathematical morphology is theoretically formulated in the framework of complete lattices and operators defined on them [29, 21]. When only the supremum or the infimum are well defined, other morphological operators can be formulated in the framework of complete semilattices [23, 22]. Both cases are considered here for images $f \in \mathcal{F}(\Omega, \mathcal{N})$.

A possible way to deal with the partial ordering problem of $\mathcal{N}$ can be founded on stochastic ordering (or stochastic dominance) [30] which is basically defined in terms of majorization of cumulative distribution functions.

However, we prefer to adopt here an information geometry approach [4], which is based on considering that the univariate Gaussian pdfs are points in a hyperbolic space $[9,3]$. More generally, Fisher geometry amounts to hyperbolic geometry of constant curvature for other location-scale families of probability distributions (Cauchy, Laplace, elliptical) $p(x ; \mu, \sigma)=$ $\frac{1}{\sigma} f\left(\frac{x-\mu}{\sigma}\right)$, where curvature depends on the dimension and the density profile $[3,14,15]$. For a deep flavor on hyperbolic geometry see [12]. There are several models representing the hyperbolic space in $\mathbb{R}^{d}, d>1$, such as the three following ones: the (Poincaré) upper half- 

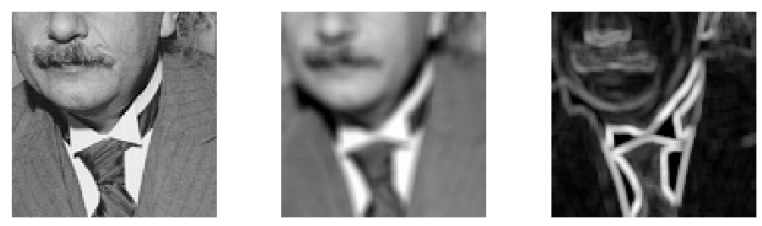

(a)

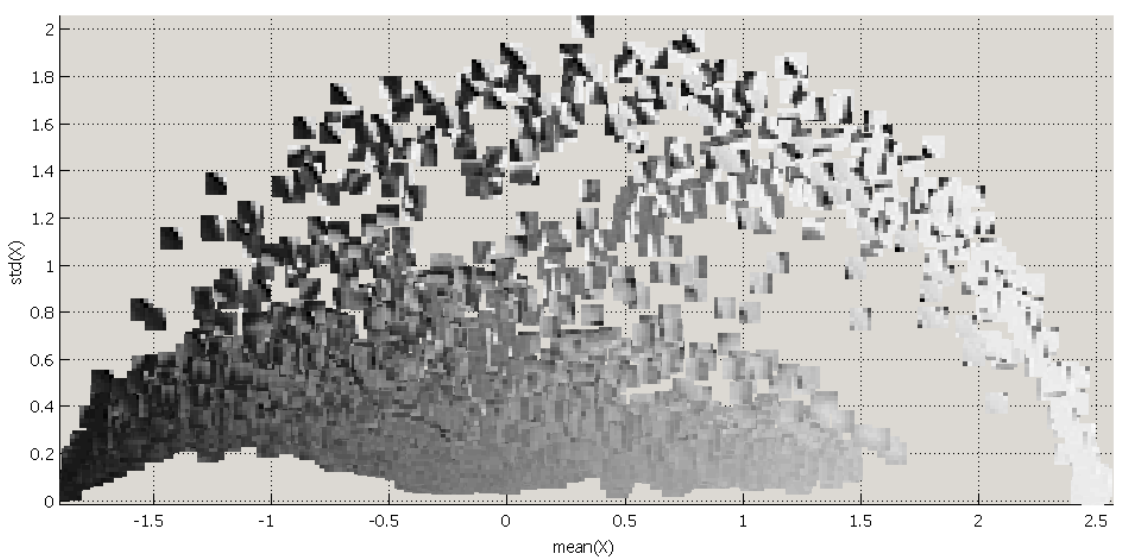

(b)

Figure 1: Parametrization of a gray scale image where each pixel is described by the mean and the variance of the intensity distribution from its centered neighboring patch of $5 \times 5$ pixels: (a) Left, original gray scale image; center, image of mean of each patch; right, image of standard deviation of each patch. (b) Visualization of the patch according to their coordinates in the space mean/std.dev.

space model $\mathcal{H}^{d}$, the Poincaré disk model $\mathcal{P}^{d}$ and the Klein disk model $\mathcal{K}^{d}$.

1. The (Poincaré) upper half-space model is the domain $\mathcal{H}^{d}=\left\{\left(x_{1}, \cdots, x_{d}\right) \in \mathbb{R}^{d} \mid x_{d}>0\right\}$ with the Riemannian metric $d s^{2}=\frac{d x_{1}^{2}+\cdots+d x_{d}^{2}}{x_{d}^{2}}$;

2. The Poincaré disk model is the domain $\mathcal{P}^{d}=\left\{\left(x_{1}, \cdots, x_{d}\right) \in \mathbb{R}^{d} \mid x_{1}^{2}+\cdots+x_{d}^{2}<1\right\}$ with the Riemannian metric $d s^{2}=4 \frac{d x_{1}^{2}+\cdots+d x_{d}^{2}}{\left(1-x_{1}^{2}-\cdots-x_{d}^{2}\right)^{2}}$;

3. The Klein disk model is the space $\mathcal{K}^{d}=\left\{\left(x_{1}, \cdots, x_{d}\right) \in \mathbb{R}^{d} \mid x_{1}^{2}+\cdots+x_{d}^{2}<1\right\}$ with the Riemannian metric $d s^{2}=\frac{d x_{1}^{2}+\cdots+d x_{d}^{2}}{1-x_{1}^{2}-\cdots-x_{d}^{2}}+\frac{\left(x_{1} d x_{1}+\cdots+x_{d} d x_{d}\right)^{2}}{\left(1-x_{1}^{2}-\cdots-x_{d}^{2}\right)^{2}}$.

These models are isomorphic between them in the sense that one-to-one correspondences can be set up between the points and lines in one model to the points and lines in the other so as to preserve the relations of incidence, betweenness and congruence. In particular, there exists an isometric mapping between any pair among these models and analytical transformations to convert from one to another are well known [12]. 


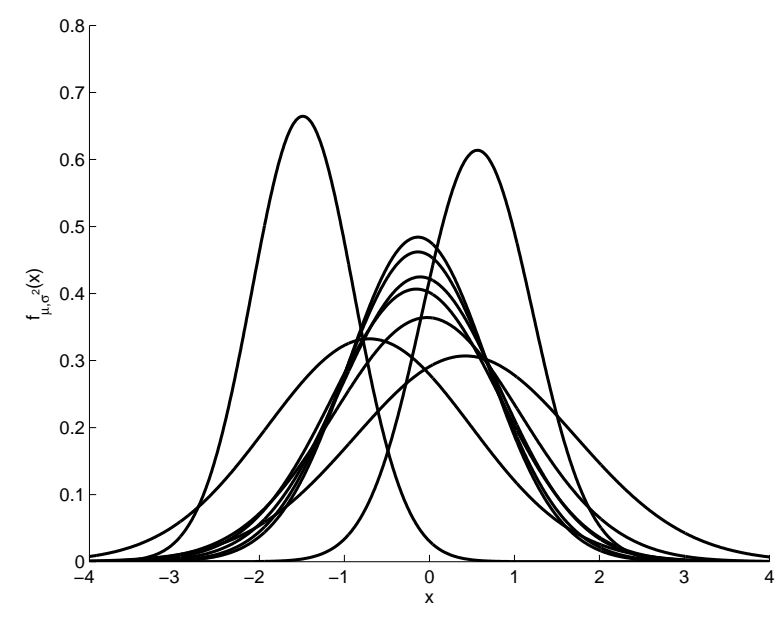

(a)

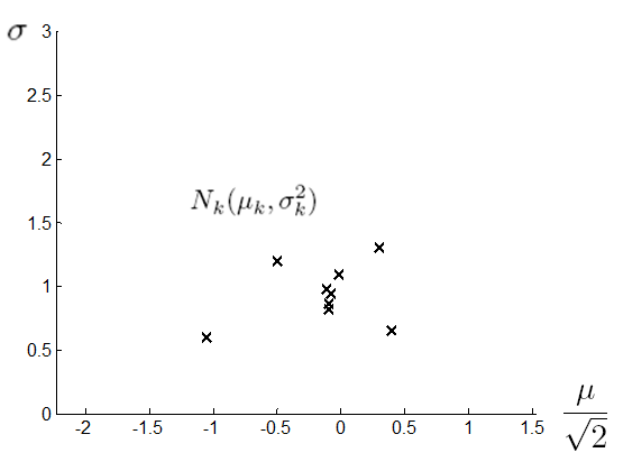

(b)

Figure 2: (a) Example of a set of nine univariate Gaussian pdfs, $N_{k}\left(\mu_{k}, \sigma_{k}^{2}\right), 1 \leq k \leq 9$. (b) Same set of Gaussian pdfs represented as points of coordinates $\left(x_{k}=\mu_{k} / \sqrt{2}, y_{k}=\sigma_{k}\right)$ in the upper-half plane.

Klein disk model has been considered for instance in computational information geometry (Voronoï diagrams, clustering, etc.) [26] and Poincaré disk model in information geometric radar processing $[5,6,7]$. In this paper, we focus on the Poincaré half-plane model, $\mathcal{H}^{2}$, which is sufficient for our practical purposes of manipulating Gaussian pdfs. Fig. 2(b) illustrates the example of a set of nine Gaussian pdfs $N_{k}\left(\mu_{k}, \sigma_{k}^{2}\right)$ represented as points of coordinates $\left(\mu_{k} / \sqrt{2}, \sigma_{k}\right)$ in the upper-half plane as follows:

$$
(\mu, \sigma) \mapsto z=\frac{\mu}{\sqrt{2}}+i \sigma
$$

The rationale behind the scaling factor $\sqrt{2}$ is given in Section 3 .

In summary, from a theoretical viewpoint, the aim of this paper is to endow $\mathcal{H}^{2}$ with partial orderings which lead to useful invariance properties in order to formulate appropriate morphological operators for images $f: \Omega \rightarrow \mathcal{H}^{2}$. This work is an extension to the conference paper [1]. The rest of the paper is organized as follows. Section 2 reminds the basics on the geometry of Poincaré half-plane model. The connection between Poincaré half-plane model of hyperbolic geometry and Fisher Information geometry of Gaussian distributions is briefly recalled in Section 3. Then, various partial orderings on $\mathcal{H}^{2}$ are studied in Section 4. Based on the corresponding complete lattice structure of $\mathcal{H}^{2}$, Section 5 presents definition of morphological operators for images on $\mathcal{F}\left(\Omega, \mathcal{H}^{2}\right)$ and its application to morphological processing univariate Gaussian distribution-valued images. Section 6 concludes the paper with the perspectives of the present work. 


\section{Geometry of Poincaré upper-half plane $\mathcal{H}^{2}$}

In complex analysis, the upper-half plane is the set of complex numbers with positive imaginary part:

$$
\mathcal{H}^{2}=\{z=x+i y \in \mathbb{C} \mid y>0\} .
$$

We also use the notation $x=\Re(z)$ and $y=\Im(z)$. The boundary of upper-half plane (called sometimes circle at infinity) is the real axis together with the infinity, i.e., $\partial \mathcal{H}^{2}=\mathbb{R} \cup \infty=$ $\{z=x+i y \mid y=0, x= \pm \infty, y=\infty\}$.

\subsection{Riemannian metric, angle and distance}

In hyperbolic geometry, the Poincaré upper-half plane model (originated with Beltrami and also known as Lobachevskii space in Soviet scientific literature) is the space $\mathcal{H}^{2}$ together with the Poincaré metric

$$
\left(g_{k l}\right)_{k, l=1,2}=\left(\begin{array}{cc}
\frac{1}{y^{2}} & 0 \\
0 & \frac{1}{y^{2}}
\end{array}\right)
$$

such that the hyperbolic arc length is given by

$$
d s^{2}=\sum_{k, l=1,2} g_{k l} d x d y=\frac{d x^{2}+d y^{2}}{y^{2}}=\frac{|d z|^{2}}{y^{2}}=y^{-1} d z y^{-1} d z^{*} .
$$

With this metric, the Poincaré upper-half plane is a complete Riemannian manifold of constant sectional curvature $K$ equal to -1 . We can consider a continuum of other hyperbolic spaces by multiplying the hyperbolic arc length (3) by a positive constant $k$ which leads to a metric of constant Gaussian curvature $K=-1 / k^{2}$. The tangent space to $\mathcal{H}^{2}$ at a point $z$ is defined as the space of tangent vectors at $z$. It has the structure of a 2-dimensional real vector space, $T_{z} \mathcal{H}^{2} \simeq \mathbb{R}^{2}$. The Riemannian metric (3) is induced by the following inner product on $T_{z} \mathcal{H}^{2}$ : for $\zeta_{1}, \zeta_{2} \in T_{z} \mathcal{H}^{2}$, with $\zeta_{k}=\left(\xi_{k}, \eta_{k}\right)$, we put

$$
\left\langle\zeta_{1}, \zeta_{2}\right\rangle_{z}=\frac{\left(\zeta_{1}, \zeta_{2}\right)}{\Im(z)^{2}}
$$

which is a scalar multiple of the Euclidean inner product $\left(\zeta_{1}, \zeta_{2}\right)=\xi_{1} \xi_{2}+\eta_{1} \eta_{2}$.

The angle $\theta$ between two geodesics in $\mathcal{H}^{2}$ at their intersection point $z$ is defined as the angle between their tangent vectors in $T_{z} \mathcal{H}^{2}$, i.e.,

$$
\cos \theta=\frac{\left\langle\zeta_{1}, \zeta_{2}\right\rangle_{z}}{\left\|\zeta_{1}\right\|_{z}\left\|\zeta_{2}\right\|_{z}}=\frac{\left(\zeta_{1}, \zeta_{2}\right)}{\sqrt{\left(\zeta_{1}, \zeta_{1}\right)} \sqrt{\left(\zeta_{2}, \zeta_{2}\right)}}
$$

We see that this notion of angle measure coincides with the Euclidean angle measure. Consequently, the Poincaré upper-half plane is a conformal model. 
The distance between two points $z_{1}=x_{1}+i y_{1}$ and $z_{2}=x_{2}+i y_{2}$ in $\left(\mathcal{H}^{2}, d s^{2}\right)$ is the function

$$
\operatorname{dist}_{\mathcal{H}^{2}}\left(z_{1}, z_{2}\right)=\cosh ^{-1}\left(1+\frac{\left(x_{1}-x_{2}\right)^{2}+\left(y_{1}-y_{2}\right)^{2}}{2 y_{1} y_{2}}\right)
$$

Distance (6) is derived from the logarithm of the cross-ratio between these two points and the points at the infinity, i.e., $\operatorname{dist}_{\mathcal{H}^{2}}\left(z_{1}, z_{2}\right)=\log D\left(z_{1}^{\infty}, z_{1}, z_{2}, z_{2}^{\infty}\right)$ where $D\left(z_{1}^{\infty}, z_{1}, z_{2}, z_{2}^{\infty}\right)=$ $\frac{z_{1}-z_{2}^{\infty}}{z_{1}-z_{1}^{\infty}} \frac{z_{2}-z_{1}^{\infty}}{z_{2}-z_{2}^{\infty}}$. To obtain their equivalence, we remind that $\cosh ^{-1}(x)=\log \left(x+\sqrt{x^{2}-1}\right)$. From this formulation it is easy to check that for two points with $x_{1}=x_{2}$ the distance is $\operatorname{dist}_{\mathcal{H}^{2}}\left(z_{1}, z_{2}\right)=\left|\log \left(\frac{y_{1}}{y_{2}}\right)\right|$.

To see that $\operatorname{dist}_{\mathcal{H}^{2}}\left(z_{1}, z_{2}\right)$ is a metric distance in $\mathcal{H}^{2}$, we first notice the argument of $\cosh ^{-1}$ always lies in $[1, \infty)$ and $\cosh (x)=\frac{e^{x}+e^{-x}}{2}$, so cosh is increasing and concave on $[0, \infty)$. Thus $\cosh ^{-1}(1)=0$ and $\cosh ^{-1}$ is increasing and concave down on $[1, \infty)$, growing logarithmically. The properties required to be a metric (non-negativity, symmetry and triangle inequality) are proven using the cross-ratio formulation of the distance.

We note that the distance from any point $z \in \mathcal{H}^{2}$ to $\partial \mathcal{H}^{2}$ is infinity.

\subsection{Geodesics}

The geodesics of $\mathcal{H}^{2}$ are the vertical lines, $V L(a)=\left\{z \in \mathcal{H}^{2} \mid \Re(z)=a\right\}$, and the semi-circles in $\mathcal{H}^{2}$ which meet the horizontal axis $\Re(z)=0$ orthogonally, $S C_{r}(a)=\left\{z \in \mathcal{H}^{2}|| z-z^{\prime} \mid=\right.$ $r$; $\Re\left(z^{\prime}\right)=a$ and $\left.\Im\left(z^{\prime}\right)=0\right\}$; see Fig $3\left(\right.$ a). Thus given any pair $z_{1}, z_{2} \in \mathcal{H}^{2}$, there is a unique geodesic connecting them, parameterized for instance in polar coordinates by the angle, i.e.,

$$
\gamma(t)=a+r e^{i t}=(a+r \cos t)+i(r \sin t), \quad \theta_{1} \leq t \leq \theta_{2}
$$

where $z_{1}=a+r e^{i \theta_{1}}$ and $z_{2}=a+r e^{i \theta_{2}}$.

A more useful expression of the geodesics involves explicitly the cartesian coordinates of the pair of points. First, given the pair $z_{1}, z_{2} \in \mathcal{H}^{2}, x_{1} \neq x_{2}$, the semi-circle orthogonal to $\mathrm{x}$ axis connecting them has a center $c=\left(a_{1 \frown 2}, 0\right)$ and a radius $r_{1 \frown 2},\left(z_{1}, z_{2}\right) \mapsto S C_{r_{1 \frown 2}}\left(a_{1 \frown 2}\right)$, where

$$
a_{1 \frown 2}=\frac{x_{2}^{2}-x_{1}^{2}+y_{2}^{2}-y_{1}^{2}}{2\left(x_{2}-x_{1}\right)} ; \quad r_{1 \frown 2}=\sqrt{\left(x_{1}-a_{1 \frown 2}\right)^{2}+y_{1}^{2}}=\sqrt{\left(x_{2}-a_{1 \frown 2}\right)^{2}+y_{2}^{2}} .
$$

Then, the unique geodesic parameterized by the length, $t \mapsto \gamma\left(z_{1}, z_{2} ; t\right), \gamma:[0,1] \rightarrow \mathcal{H}^{2}$ joining two points $z_{1}=x_{1}+i y_{1}$ and $z_{2}=x_{2}+i y_{2}$ such as $\gamma\left(z_{1}, z_{2} ; 0\right)=z_{1}$ and $\gamma\left(z_{1}, z_{2} ; 1\right)=z_{2}$ is given by

$$
\gamma\left(z_{1}, z_{2} ; t\right)= \begin{cases}x_{1}+i e^{\xi t+t_{0}} & \text { if } x_{1}=x_{2} \\ {\left[r \tanh \left(\xi t+t_{0}\right)+a\right]+i\left[\frac{r}{\cosh \left(\xi t+t_{0}\right)}\right]} & \text { if } x_{1} \neq x_{2}\end{cases}
$$

with $a$ and $r$ given in (8) and where for $x_{1}=x_{2}, t_{0}=\log \left(y_{1}\right), \xi=\log \frac{y_{2}}{y_{1}}$ and for $x_{1} \neq x_{2}$

$$
t_{0}=\cosh ^{-1}\left(\frac{r}{y_{1}}\right)=\sinh ^{-1}\left(\frac{x_{1}-a}{y_{1}}\right), \xi=\log \left(\frac{y_{1}}{y_{2}} \frac{r+\sqrt{r^{2}-y_{2}^{2}}}{r+\sqrt{r^{2}-y_{1}^{2}}}\right) .
$$




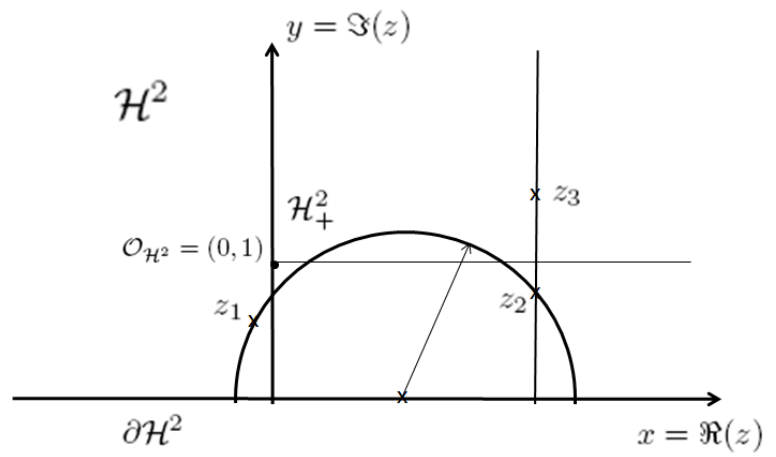

(a)

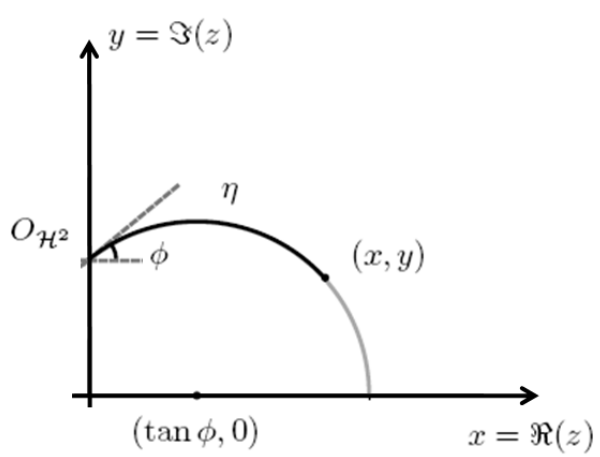

(b)

Figure 3: (a) Geodesics of $\mathcal{H}^{2}: z_{1}$ and $z_{2}$ are connected by a unique semi-circle; the geodesic between $z_{2}$ and $z_{3}$ is a segment of vertical line. (b) Hyperbolic polar coordinates.

If we take the parameterized smooth curve $\gamma(t)=x(t)+i y(t)$, where $x(t)$ and $y(t)$ are continuously differentiable for $b \leq t \leq c$, then the hyperbolic length along the curve is determined by integrating the metric (3) as:

$$
L(\gamma)=\int_{\gamma} d s=\int_{a}^{b}|\dot{\gamma}(t)|_{\gamma} d t=\int_{a}^{b} \frac{\sqrt{\dot{x}(t)^{2}+\dot{y}(t)^{2}}}{y(t)} d t=\int_{a}^{b} \frac{|\dot{z}(t)|}{y(t)} d t .
$$

Note that this expression is independent from the parameter choice. Hence, using the polar angle parametrization (7), we obtain an alternative expression of the geodesic distance given

$$
\operatorname{dist}_{\mathcal{H}^{2}}\left(z_{1}, z_{2}\right)=\inf _{\gamma} L(\gamma)=\int_{\theta_{1}}^{\theta_{2}} \frac{r}{r \sin t} d t=\left|\log \left(\cot \frac{\theta_{2}}{2}\right)-\log \left(\cot \frac{\theta_{1}}{2}\right)\right|
$$

which is independent of $r$ and consequently, as described below, dilation is an isometry.

Remark. Interpolation between two univariate normal distributions. Using the closedform expression of geodesics $t \mapsto \gamma\left(z_{1}, z_{2} ; t\right)$, given in (9), it is possible to compute the average univariate Gaussian pdf between $N\left(\mu_{1}, \sigma_{1}^{2}\right)$ and $N\left(\mu_{2}, \sigma_{2}^{2}\right)$, with $\left(\mu_{k}=\sqrt{2} x_{k}, \sigma_{k}=y_{k}\right)$, by taking $t=0.5$. More generally, we can interpolate a series of distributions between them by discretizing $t$ between 0 and 1. An example of such a method is given in Fig. 4. We note in particular that the average Gaussian pdf can have a variance bigger than $\sigma_{1}^{2}$ and $\sigma_{2}^{2}$. We note also that, due to the "logarithmic scale" of imaginary axis, equally spaces points in $t$ do not have equal Euclidean arc-length in the semi-circle.

\subsection{Hyperbolic polar coordinates}

The position of point $z=x+i y$ in $\mathcal{H}^{2}$ can be given either in terms of Cartesian coordinates $(x, y)$ or by means of polar hyperbolic coordinates $(\eta, \phi)$, where $\eta$ represents the distance of 


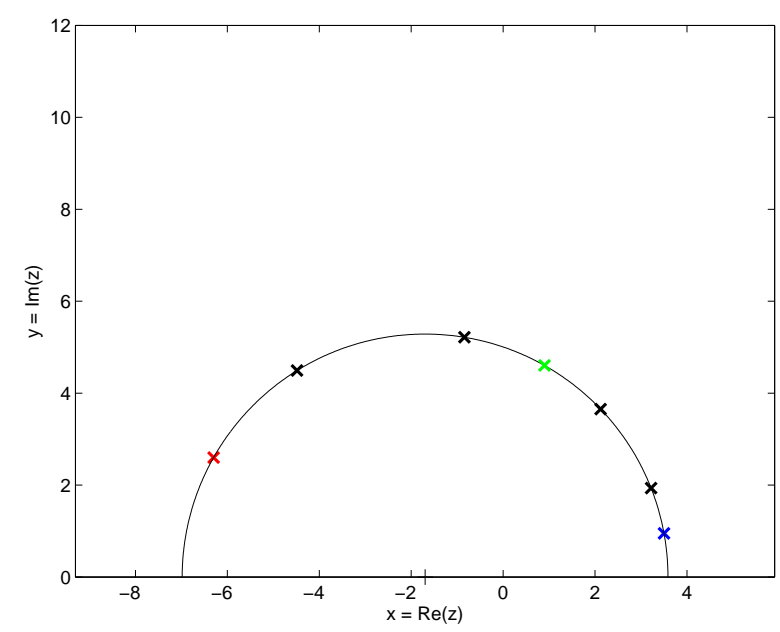

(a)

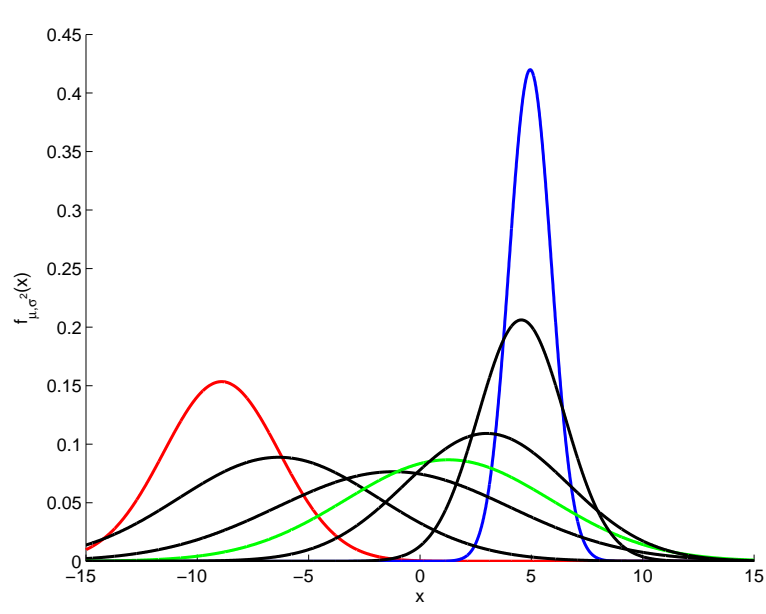

(b)

Figure 4: (a) Example of interpolation of 5 points in $\mathcal{H}^{2}$ between points $z_{1}=-6.3+i 2.6$ (in red) and $z_{2}=3.5+i 0.95$ (in blue) using their geodesic $t \mapsto \gamma\left(z_{1}, z_{2} ; t\right)$, with $t=0.2,0.4$, $0.5,0.6,0.8$. The average point (in green) just corresponds to $\gamma\left(z_{1}, z_{2} ; 0.5\right)=0.89+i 4.6$. (b) Original (in red and blue) univariate Gaussian pdfs and corresponding interpolated ones.

the point from the origin $\mathcal{O}_{\mathcal{H}^{2}}=(0,1)$ and $\phi$ represents the slope of the tangent in $\mathcal{O}_{\mathcal{H}^{2}}$ to the geodesic (i.e., semi-circle) joining the point $(x, y)$ with the origin. The formulas which relate the hyperbolic coordinates $(\eta, \phi)$ to the Cartesian ones $(x, y)$ are [11]

$$
\left\{\begin{array} { l l } 
{ x = \frac { \operatorname { s i n h } \eta \operatorname { c o s } \phi } { \operatorname { c o s h } \eta - \operatorname { s i n h } \eta \operatorname { s i n } \phi } , } & { \eta > 0 } \\
{ y = \frac { 1 } { \operatorname { c o s h } \eta - \operatorname { s i n h } \eta \operatorname { s i n } \phi } , } & { - \frac { \pi } { 2 } < \phi < \frac { \pi } { 2 } }
\end{array} \quad \left\{\begin{array}{l}
\eta=\operatorname{dist}_{\mathcal{H}^{2}}\left(\mathcal{O}_{\left.\mathcal{H}^{2}, z\right)}\right. \\
\phi=\arctan \frac{x^{2}+y^{2}-1}{2 x}
\end{array}\right.\right.
$$

We notice that the center of the geodesic passing through $(x, y)$ from $\mathcal{O}_{\mathcal{H}^{2}}$ has Cartesian coordinates given by $(\tan \phi, 0)$; see Fig $3(\mathrm{~b})$.

\subsection{Invariance and isometric symmetry}

Let the projective special linear group defined by $\operatorname{PSL}(2, \mathbb{R})=\operatorname{SL}(2, \mathbb{R}) /\{ \pm \mathrm{I}\}$ where the special linear group $\operatorname{SL}(2, \mathbb{R})$ consists of $2 \times 2$ matrices with real entries which determinant equals +1 , i.e.,

$$
g \in \mathrm{SL}(2, \mathbb{R}): g=\left(\begin{array}{ll}
a & b \\
c & d
\end{array}\right), a d-b c=1
$$

and I denotes the identity matrix. This defines the group of Möbius transformations $M_{g}$ : $\mathcal{H}^{2} \rightarrow \mathcal{H}^{2}$ by setting for each $g \in \mathrm{SL}(2, \mathbb{R})$,

$$
z \mapsto M_{g}(z)=\left(\begin{array}{ll}
a & b \\
c & d
\end{array}\right) \cdot z=\frac{a z+b}{c z+d}=\frac{a c|z|^{2}+b d+(a d+b c) \Re(z)+i \Im(z)}{|c z+d|^{2}},
$$


such that $\Im\left(M_{g}(z)\right)=(y(a d-b c)) /\left((c x+d)^{2}+(c y)^{2}\right)>0$. The inverse map is easily computed, i.e., $z \mapsto M_{g}^{-1}(z)=(d z-b) /(-c z+a)$. Since Möbius transformations are well defined in $\mathcal{H}^{2}$ and map $\mathcal{H}^{2}$ to $\mathcal{H}^{2}$ homeomorphically.

The Lie group $\operatorname{PSL}(2, \mathbb{R})$ acts on the upper half-plane by preserving the hyperbolic distance, i.e.,

$$
\operatorname{dist}_{\mathcal{H}^{2}}\left(M_{g}\left(z_{1}\right), M_{g}\left(z_{2}\right)\right)=\operatorname{dist}_{\mathcal{H}^{2}}\left(z_{1}, z_{2}\right), \forall g \in \mathrm{SL}(2, \mathbb{R}), \forall z_{1}, z_{2} \in \mathcal{H}^{2}
$$

This includes three basic types of transformations: (1) translations $z \mapsto z+\alpha, \alpha \in \mathbb{R}$; (2) scaling $z \mapsto \beta z, \beta \in \mathbb{R} \backslash 0$; (3) inversion $z \mapsto z^{-1}$. More precisely, any generic Möbius transformation $M_{g}(z), c \neq 0$, can be decomposed into the following four maps: $f_{1}=z+d / c$, $f_{2}=-1 / z, f_{3}=z(a d-b c) / c^{2}, f_{4}=z+a / c$ such that $M_{g}(z)=\left(f_{1} \circ f_{2} \circ f_{3} \circ f_{4}\right)(z)$. If $c=0$, we have $M_{g}(z)=\left(h_{1} \circ h_{2} \circ h_{3}\right)(z)$ where $h_{1}(z)=a z, h_{2}(z)=z+b, h_{3}(z)=z / d$. It can be proved that Möbius transformations take circles to circles. Hence, given a circle in the complex plane $\mathbb{C}$ of radius $r$ and center $c$, denoted by $C_{r}(c)$, we have its following mappings [27]: a translation $z \mapsto z+\alpha$, such as the functions $f_{1}, f_{4}$ and $h_{2}$ maps $C_{r}(c)$ to $C_{r}(c+\alpha)$; a scaling $z \mapsto \beta z$, such as the functions $f_{3}, h_{1}$ and $h_{3}$, maps $C_{r}(c)$ to $C_{\beta r}(\beta c)$; for inversion $z \mapsto z^{-1}, C_{r}(c)$ maps to $C_{r /|c z|}(-1 / c)$.

Let $H \in \mathcal{H}^{2}$ be a geodesic of the upper half-plane, which is described uniquely by its endpoints in $\partial \mathcal{H}^{2}$, there exists a Möbius transformation $M_{g}$ such that $M_{g}$ maps $H$ bijectively to the imaginary axis, i.e., $V L(0)$. If $H$ is the vertical line $V L(a)$, the transformation is the translation $z \mapsto M_{g}(z)=z-a$. If $H$ is the semi-circle $S C_{r}(a)$ with endpoints in real axis being $\zeta_{-}, \zeta_{+} \in \mathbb{R}$, where $\zeta_{-}=a-r$ and $\zeta_{+}=a+r$, the map is given by $M_{g}(z)=\frac{z-\zeta_{-}}{z-\zeta_{+}}$, such that $M_{g}\left(\zeta_{-}\right)=0, M_{g}\left(\zeta_{+}\right)=\infty$ and $M_{g}(a+i r)=i$.

The unit-speed geodesic going up vertically, through the point $z=i$ is given by

$$
\gamma(t)=\left(\begin{array}{cc}
e^{t / 2} & 0 \\
0 & e^{-t / 2}
\end{array}\right) \cdot i=i e^{t}
$$

Because $\operatorname{PSL}(2, \mathbb{R})$ acts transitively by isometries of the upper half-plane, this geodesic is mapped into other geodesics through the action of $\operatorname{PSL}(2, \mathbb{R})$. Thus, the general unit-speed geodesic is given by

$$
\gamma(t)=\left(\begin{array}{ll}
a & b \\
c & d
\end{array}\right)\left(\begin{array}{cc}
e^{t / 2} & 0 \\
0 & e^{-t / 2}
\end{array}\right) \cdot i=\frac{a i e^{t}+b}{c i e^{t}+d}
$$

\subsection{Hyperbolic circles and balls}

Let consider an Euclidean circle of center $c=\left(x_{c}, y_{c}\right) \in \mathcal{H}^{2}$ and radius $r$ in the upper-half plane, defined as $C_{r}(c)=\left\{z \in \mathcal{H}^{2} \mid \sqrt{\left(x_{c}-a\right)^{2}+\left(y_{c}-b\right)^{2}}=r\right\}$, such that it is contained in the upper-half plane, i.e., $C_{r}(c) \subset \mathcal{H}^{2}$. The corresponding hyperbolic circle $C_{\mathcal{H}^{2}, r_{h}}\left(c_{h}\right)$ 
$\left\{z \in \mathcal{H}^{2} \mid \operatorname{dist}_{\mathcal{H}^{2}}\left(c_{h}, z\right)=r_{h}\right\}$ is geometrically equal to $C_{r}(c)$ but its hyperbolic center and radius are given by

$$
c_{h}=\left(x_{c}, \sqrt{y_{c}^{2}-r^{2}}\right) ; \quad r_{h}=\tanh ^{-1}\left(\frac{r}{y_{c}}\right) .
$$

We note that the hyperbolic center is always below the Euclidean center. The inverse equations are

$$
c=\left(x_{c}=x_{h}, y_{c}=y_{h} \cosh r_{h}\right) ; \quad r=y_{h} \sinh r_{h} .
$$

Naturally, the hyperbolic ball of center $c_{h}$ and radius $r_{h}$ is defined by $B_{\mathcal{H}^{2}, r_{h}}\left(c_{h}\right)\{z \in$ $\left.\mathcal{H}^{2} \mid \operatorname{dist}_{\mathcal{H}^{2}}\left(c_{h}, z\right) \leq r_{h}\right\}$. Let us consider a hyperbolic ball centered at the origin $B_{\mathcal{H}^{2}, r_{h}}(0,1)$, parameterized by its boundary curve $\partial B$ in Euclidean coordinates:

$$
x=r \cos \theta ; \quad y=b+r \sin \theta
$$

where using (12), we have $b=\cosh r_{h}$ and $r=\sinh r_{h}$. The length of the boundary and area of this ball are respectively given by [32]:

$$
\begin{aligned}
L(\partial B) & =\int_{0}^{2 \pi} \frac{r}{b+r \sin \theta} d \theta=2 \pi \sinh r_{h}, \\
\operatorname{Area}(B) & =\iint_{B} \frac{d x d y}{y^{2}}=\oint_{\gamma} \frac{d x}{y}=2 \pi\left(\cosh r_{h}-1\right) .
\end{aligned}
$$

Comparing the values of an Euclidean ball which has area $\pi r_{h}^{2}$ and length of its boundary circle $2 \pi r_{h}$, and considering the Taylor series $\sinh r_{h}=r_{h}+\frac{r_{h}^{3}}{3 !}+\frac{r_{h}^{5}}{5 !}+\cdots$ and $\cosh r_{h}=$ $1+\frac{r_{h}^{2}}{2 !}+\frac{r_{h}^{4}}{4 !}+\cdots$, one can note that the hyperbolic space is much larger than Euclidean one. Curvature is defined through derivatives of the metric, but the fact that infinitesimally the hyperbolic ball grows faster than the Euclidean balls, a measure of the curvature of the space at the origin $(0,1)$ can be used [32]: $K=\lim _{r_{h} \rightarrow 0} \frac{3\left[2 \pi r_{h}-L(\partial B)\right]}{\pi r_{h}^{3}}=-1$. Since there is an isometry that maps the neighborhood of any point to the neighborhood of the origin, the curvature of hyperbolic space is identically constant to -1 .

Remark. Minimax center in $\mathcal{H}^{2}$. Finding the smallest circle that contains the whole set of points $x_{1}, x_{2} \cdots, x_{N}$ in the Euclidean plane is a classical problem in computational geometry, called the minimum enclosing circle $M E C$. It is also relevant its statistical estimation since the unique center of the circle $c^{\infty}$ (called 1-center or minimax center) is defined as the $L^{\infty}$ center of mass, i.e., for $\mathbb{R}^{2}, c^{\infty}=\arg \min _{x \in \mathbb{R}^{2}} \max _{1 \leq i \leq N}\left\|x_{i}-x\right\|_{2}$. Computing the smallest enclosing sphere in Euclidean spaces is intractable in high dimensions, but efficient approximation algorithms have been proposed. The Bădoiu and Clarkson algorithm [8] leads to a fast and simple approximation (of known precision $\epsilon$ after a given number of iterations $\left\lceil\frac{1}{\epsilon^{2}}\right\rceil$ using the notion of core-set, but independent from dimensionality $n$ ). The computation of the minimax center is particularly relevant in information geometry (smallest enclosing information disk [25]) and has been considered for hyperbolic models such as the Klein disk, 
using a Riemannian extension of Bădoiu and Clarkson algorithm [2], which only requires a closed-form of the geodesics. Fig. 5 depicts an example of minimax center computation using Bădoiu and Clarkson algorithm for a set of univariate Gaussian pdfs represented in $\mathcal{H}^{2}$. We note that, using this property of circle preservation, the computation of the minimal enclosing hyperbolic circle of a given set of points $Z=\left\{z_{k}\right\}_{1 \leq k \leq K}, z_{k} \in \mathcal{H}^{2}$, denoted $\operatorname{MEC}_{\mathcal{H}^{2}}(Z)$ is equivalent to computing the corresponding minimal enclosing circle $M E C(Z)$ if and only if we have $\operatorname{MEC}(Z) \subset \mathcal{H}^{2}$. This is the case for the example given in Fig. 5.

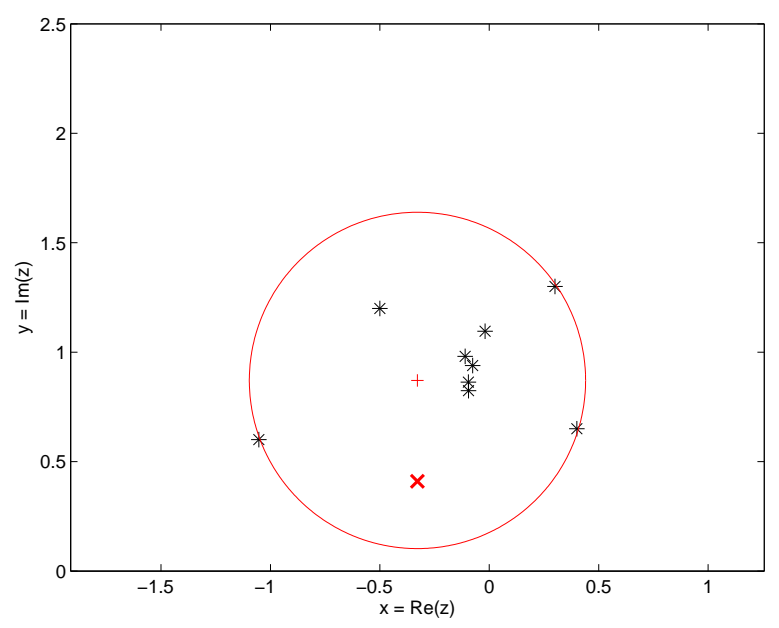

(a)

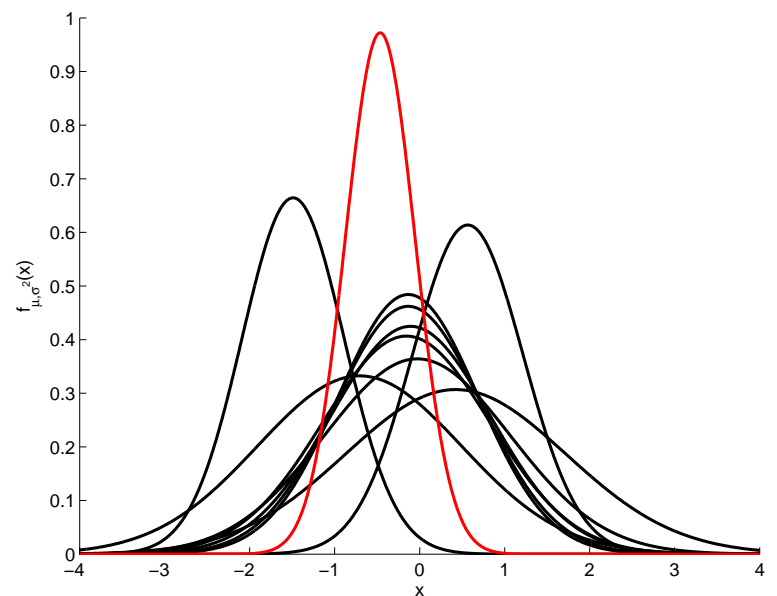

(b)

Figure 5: (a) Example of minimax center $\left(x_{h}, y_{h}\right)($ red $\times)$ of a set of nine points $Z=\left\{z_{k}\right\}_{1 \leq k \leq 9}$ in $\mathcal{H}^{2}$ (original points $*$ in black), the minimal enclosing circle $M E C_{\mathcal{H}^{2}}(Z)$ is also depicted (in red). (b) Corresponding minimax center Gaussian set $N\left(\mu=\sqrt{2} x_{h}, \sigma^{2}=y_{h}^{2}\right)$ of nine univariate Gaussian pdfs, $N_{k}\left(\mu_{k}, \sigma_{k}^{2}\right), 1 \leq k \leq 9$.

\section{Fisher information metric and $\alpha$-order entropy metric of univariate normal distributions}

In information geometry, the Fisher information metric is a particular Riemannian metric which can be associated to a smooth manifold whose points are probability measures defined on a common probability space $[3,4]$. It can be obtained as the infinitesimal form of the Kullback-Leibler divergence (relative entropy). An alternative formulation is obtained by computing the negative of the Hessian of the Shannon entropy.

Given an univariate probability distribution $p(x \mid \theta), x \in X$, it can be viewed as a point on a statistical manifold with coordinates given by $\theta=\left(\theta_{1}, \theta_{2}, \cdots, \theta_{n}\right)$. The Fisher information 
matrix then takes the form:

$$
g_{k l}(\theta)_{k, l=1,2}=\int_{X} \frac{\partial \log p(x, \theta)}{\partial \theta_{k}} \frac{\partial \log p(x, \theta)}{\partial \theta_{l}} p(x, \theta) d x .
$$

The corresponding positive definite form

$$
d s^{2}(\theta)=\sum_{k, l=1}^{n} g_{k l}(\theta) d \theta_{k} d \theta_{l}
$$

is defined as the Fisher information metric. In the univariate Gaussian distributed case $p(x \mid \theta) \equiv N\left(\mu, \sigma^{2}\right)$, we have in particular $\theta=(\mu, \sigma)$ and it can be easily deduced that the Fisher information matrix is

$$
\left(g_{k l}(\mu, \sigma)\right)=\left(\begin{array}{cc}
\frac{1}{\sigma^{2}} & 0 \\
0 & \frac{2}{\sigma^{2}}
\end{array}\right)
$$

and the corresponding metric is

$$
d s^{2}((\mu, \sigma))=\frac{d \mu^{2}+2 d \sigma^{2}}{\sigma^{2}}=2 \sigma^{-2}\left(\frac{d \mu^{2}}{\sqrt{2}}+d \sigma^{2}\right) .
$$

Therefore, the Fisher information geometry of univariate normal distribution is essentially the geometry of the Poincaré upper-half plane with the following change of variables:

$$
x=\mu / \sqrt{2}, \quad y=\sigma
$$

Hence, given two univariate Gaussian pdfs $N\left(\mu_{1}, \sigma_{1}^{2}\right)$ and $N\left(\mu_{2}, \sigma_{2}^{2}\right)$, the Fisher distance between them, dist ${ }_{\text {Fisher }}: \mathcal{N} \times \mathcal{N} \rightarrow \mathbb{R}_{+}$, defined from the Fisher information metric is given by $[9,14]$ :

$$
\operatorname{dist}_{\text {Fisher }}\left(\left(\mu_{1}, \sigma_{1}^{2}\right),\left(\mu_{2}, \sigma_{2}^{2}\right)\right)=\sqrt{2} \operatorname{dist}_{\mathcal{H}^{2}}\left(\frac{\mu_{1}}{\sqrt{2}}+i \sigma_{1}, \frac{\mu_{2}}{\sqrt{2}}+i \sigma_{2}\right)
$$

The change of variable also involves that the geodesics in the hyperbolic Fisher space of normal distributions are half-lines and half-ellipses orthogonal at $\sigma=0$, with eccentricity $1 / \sqrt{2}$.

The canonic approach can be generalized according to Burbea and Rao geometric framework [9], which is based on replacing the Shannon entropy by the notion of $\alpha$-order entropy, which associated Hessian metric leads to an extended large class of information metric geometries. Focussing on the particular case of univariate normal distributions, $p(x \mid \theta) \equiv N\left(\mu, \sigma^{2}\right)$, we consider again points in the upper half-plane, $z=x+i y \in \mathcal{H}^{2}$ and for a given $\alpha>0$ the $\alpha$-order entropy metric is given by [9]:

$$
\left\{\begin{array}{l}
x=[A(\alpha)]^{-1 / 2} \mu, \quad y=\sigma \\
d s_{\alpha}=B(\alpha) y^{-(\alpha+1)}\left(d x^{2}+d y^{2}\right)
\end{array}\right.
$$




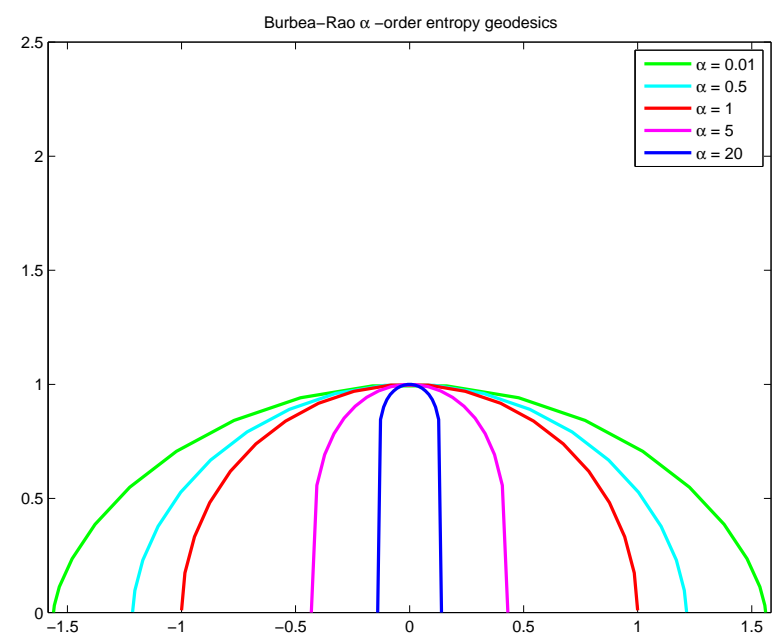

Figure 6: Examples of the geodesics from the Burbea-Rao $\alpha$-order entropy metric obtained using (20) and (21) for $a=0, r=1$ and $\alpha=0.01,0.5,1,5$ and 20.

where

$$
A(\alpha)=\left(\alpha^{1 / 2}-\alpha^{-1 / 2}\right)^{2}+2 \alpha^{-1} ; \quad B(\alpha)=\alpha^{-3 / 2}(2 \pi)^{(1-\alpha) / 2} A(\alpha) ; \quad \alpha>0 .
$$

The metric in (18) constitutes a Kähler metric on $\mathcal{H}^{2}$ and when $\alpha=1$ reduces to the Poincaré metric (3). Its Gaussian curvature is $K_{\alpha}(z)=-(\alpha+1)[B(\alpha)]^{-1} y^{\alpha-1}$; being always negative (hyperbolic geometry). In particular, for $\alpha=1$ we recover the particular constant case $K_{1}(z)=-1$.

The geodesics of the Burbea-Rao $\alpha$-order entropy metric can be written in its parametric polar form as [9]:

$$
\begin{gathered}
\gamma(\theta)=x(\theta)+i y(\theta), \quad 0<\theta<\pi, \text { with } \\
\left\{\begin{array}{l}
x(\theta)=a+r^{1 / \beta} F_{1 / \beta}(\theta), \\
y(\theta)=r^{1 / \beta} \sin ^{1 / \beta} \theta
\end{array}\right.
\end{gathered}
$$

where

$$
\begin{aligned}
& \beta=(\alpha+1) / 2, \quad r>0, \quad a \in \mathbb{R}, \\
& F_{\gamma}(\theta)=-\gamma \int_{\pi / 2}^{\theta} \sin ^{\gamma} t d t .
\end{aligned}
$$

Fig. 6 shows examples of the geodesics from the Burbea-Rao $\alpha$-order entropy metric for $a=0, r=1$ and $\alpha=0.01,0.5,1,5$ and 20 .

By integration of the metric, it is obtained the Burbea-Rao $\alpha$-order entropy geodesic distance for $z_{1}, z_{2} \in \mathcal{H}^{2}[9]$ :

$$
\operatorname{dist}_{\mathcal{H}^{2}}\left(z_{1}, z_{2} ; \alpha\right)=\frac{2 \sqrt{B(\alpha)}}{|1-\alpha|}\left|\frac{x_{1}-x_{2}}{r}+y_{1}^{(1-\alpha) / 2} \sqrt{1-r^{-2} y_{1}^{\alpha+1}}-y_{2}^{(1-\alpha) / 2} \sqrt{1-r^{-2} y_{2}^{\alpha+1}}\right| \text {, }
$$


which unfortunately depends on the value of $r$. This quantity should be determined by solving a system of three nonlinear equations for the unknown variables $\theta_{1}, \theta_{2}$ and $r$ :

$$
\left\{\begin{array}{l}
x_{1}-x_{2}=r^{1 / \beta}\left(F_{1 / \beta}\left(\theta_{1}\right)-F_{1 / \beta}\left(\theta_{2}\right)\right) \\
y_{1}=r^{1 / \beta} \sin ^{1 / \beta} \theta_{1} \\
y_{2}=r^{1 / \beta} \sin ^{1 / \beta} \theta_{2}
\end{array}\right.
$$

An alternative solution to compute a closed form distance between two univariate normal distributions $N\left(\mu_{1}, \sigma_{1}^{2}\right)$ and $N\left(\mu_{2}, \sigma_{2}^{2}\right)$ according to the Burbea-Rao $\alpha$-deformed geometry is based on the $\alpha$-order Hellinger distance [9]:

$$
\begin{gathered}
\operatorname{dist}_{\text {Hellinger }}\left(\left(\mu_{1}, \sigma_{1}^{2}\right),\left(\mu_{2}, \sigma_{2}^{2}\right) ; \alpha\right)= \\
\frac{2(2 \pi)^{(1-\alpha) / 4}}{\alpha^{5 / 4}}\left[\left(\sigma_{1}^{(1-\alpha) / 2}-\sigma_{2}^{(1-\alpha) / 2}\right)^{2}+2\left(\sigma_{1} \sigma_{2}\right)^{(1-\alpha) / 2}\left(1-\left(\frac{2 \sigma_{1} \sigma_{2}}{\sigma_{1}^{2}+\sigma_{2}^{2}}\right)^{1 / 2} \exp \left(\frac{-\alpha\left(\mu_{1}-\mu_{2}\right)^{2}}{4\left(\sigma_{1}^{2}+\sigma_{2}^{2}\right)}\right)\right)\right]^{1 / 2} .
\end{gathered}
$$

In particular, when $\alpha=1$ this formula reduces to

$$
\operatorname{dist}_{\text {Hellinger }}\left(\left(\mu_{1}, \sigma_{1}^{2}\right),\left(\mu_{2}, \sigma_{2}^{2}\right)\right)=2^{3 / 2}\left(1-\left(\frac{2 \sigma_{1} \sigma_{2}}{\sigma_{1}^{2}+\sigma_{2}^{2}}\right)^{1 / 2} \exp \left(\frac{-\left(\mu_{1}-\mu_{2}\right)^{2}}{4\left(\sigma_{1}^{2}+\sigma_{2}^{2}\right)}\right)\right)^{1 / 2}
$$

\section{Endowing $\mathcal{H}^{2}$ with partial ordering and its complete (inf- semi)lattice structures}

The notion of ordering invariance in the Poincaré upper-half plane was considered in the Soviet literature $[19,20]$. Ordering invariance with respect to simple transitive subgroup $T$ of the group of motions was studied, i.e., group $T$ consists of transformations $t$ of the form:

$$
z=x+i y \mapsto z^{\prime}=(\lambda x+\alpha)+i \lambda y
$$

where $\lambda>0$ and $\alpha$ are real numbers. We named $T$ the Guts group. We note that $T$ is just the composition of a translation and a scaling in $\mathcal{H}^{2}$, and consequently, $T$ is an isometric group (see Section 2.4).

Nevertheless, up to the best of our knowledge, the formulation of partial orders on Poincaré upper-half plane has not been widely studied. We introduce here partial orders in $\mathcal{H}^{2}$ and study invariance properties to transformations of Guts group or to other subgroups of $\mathrm{SL}(2, \mathbb{R})$ (Möbius transformations).

\subsection{Upper half-plane product ordering}

A real vector space $E$ on which a partial order $\leq$ is given (reflexive, transitive, antisymmetric) is called an ordered vector space if (i) $x, y, z \in E$ and $x \leq y$ implies $x+z \leq y+z$; (ii) $x, y \in E$, $0 \leq \lambda \in \mathbb{R}$, and $x \leq y$ implies $\lambda x \leq \lambda y$. Let us consider that the partial order $\leq$ is the product 
order. An element $x \in E$ with $x \geq 0$ (it means that all the vector components are positive) is said to be positive. The set $E_{+}=\{x \in E \mid x \geq 0\}$ for all positive elements is called the cone of positive elements. It turns out that the order of an ordered vector space is determined by the set of positive elements. Let $E$ be a vector space and $C \subset E$ a cone. Then, $x \leq y$ if $x-y \in C$ defines an order on $E$ such that $E$ is an ordered vector space with $E_{+}=C$. The notion of partially ordered vector space is naturally extended to partially ordered groups [18]. An ordered vector space $E$ is called a vector lattice $(E, \leq)$ if $\forall x, y \in E$ there exists the joint (supremum or least upper bound) $x \vee y=\sup (x, y) \in E$ and the meet (infimum or greatest lower bound) $x \wedge y=\inf (x, y) \in E$. A vector lattice is also called a Riesz space.

Thus, we can introduce a similar order structure in $\mathcal{H}^{2}$ as a product order of $\mathbb{R} \times \mathbb{R}_{+}$. To achieve this goal, we need to define, on the one hand, the equivalent of ordering preserving linear combination. More precisely, given three points $z_{1}, z_{2}, z_{3} \in \mathcal{H}^{2}$ and a scalar positive number $0 \leq \lambda \in \mathbb{R}$ we say that

$$
z_{1} \leq_{\mathcal{H}^{2}} z_{2} \text { implies } \lambda \odot z_{1} \boxplus z_{3} \leq_{\mathcal{H}^{2}} \lambda \boxminus z_{2} \boxplus z_{3},
$$

where we have introduced the following pair of operations in $\mathcal{H}^{2}$ :

$$
\lambda \boxminus z=\lambda x+i y^{\lambda} \text { and } z_{1} \boxplus z_{2}=\left(x_{1}+x_{2}\right)+i\left(y_{1} y_{2}\right) .
$$

On the other hand, the corresponding partial ordering $\leq_{\mathcal{H}^{2}}$ will be determined by the positive cone in $\mathcal{H}^{2}$ defined by $\mathcal{H}_{+}^{2}=\left\{z \in \mathcal{H}^{2} \mid x \geq 0\right.$ and $\left.y \geq 1\right\}$, i.e.,

$$
z_{1} \leq_{\mathcal{H}^{2}} z_{2} \Leftrightarrow z_{2} \boxminus z_{1} \in \mathcal{H}_{+}^{2},
$$

with $z_{2} \boxminus z_{1}=\left(x_{2}-x_{1}\right)+i\left(y_{2}^{-1} y_{1}\right)$. According to this partial ordering the corresponding supremum and infimum for any pair of points $z_{1}$ and $z_{2}$ in $\mathcal{H}^{2}$ are formulated as follows

$$
\begin{aligned}
z_{1} \vee_{\mathcal{H}^{2}} z_{2} & =\left(x_{1} \vee x_{2}\right)+i \exp \left(\log \left(y_{1}\right) \vee \log \left(y_{2}\right)\right), \\
z_{1} \wedge \mathcal{H}^{2} z_{2} & =\left(x_{1} \wedge x_{2}\right)+i \exp \left(\log \left(y_{1}\right) \wedge \log \left(y_{2}\right)\right) .
\end{aligned}
$$

Therefore $\mathcal{H}^{2}$ endowed with partial ordering (25) is a complete lattice, but it is not bounded since the greatest (or top) and least (or bottom) elements are in the boundary $\partial \mathcal{H}^{2}$. We also have a duality between supremum and infimum, i.e.,

$$
z_{1} \vee_{\mathcal{H}^{2}} z_{2}=\complement\left(\complement z_{1} \wedge_{\mathcal{H}^{2}} \complement z_{2}\right) ; \quad z_{1} \wedge_{\mathcal{H}^{2}} z_{2}=\complement\left(\complement z_{1} \vee_{\mathcal{H}^{2}} \complement z_{2}\right)
$$

with respect to the following involution

$$
z \mapsto \complement z=(-1) \odot z=-x+i y^{-1}
$$

We easily note that, in fact, $\exp \left(\log \left(y_{1}\right) \vee \log \left(y_{2}\right)\right)=y_{1} \vee y_{2}$ and similarly for the infimum, since the logarithm is an isotone mapping (i.e., monotone increasing) and therefore orderpreserving. Therefore, the partial ordering $\leq_{\mathcal{H}^{2}}$ does not involve any particular structure for 
$\mathcal{H}^{2}$ and does not take into account the Riemannian nature of the upper half plane. According to that, we note also that the partial ordering $\leq_{\mathcal{H}^{2}}$ is invariant to the Guts group of transforms, i.e.,

$$
z_{1} \leq_{\mathcal{H}^{2}} z_{2} \Leftrightarrow T\left(z_{1}\right) \leq_{\mathcal{H}^{2}} T\left(z_{2}\right)
$$

\subsection{Upper half-plane symmetric ordering}

Let us consider a symmetrization of the product ordering with respect to the origin in the upper half-plane. Given any pair of points $z_{1}, z_{2} \in \mathcal{H}^{2}$, we define the upper half-plane symmetric ordering as

$$
z_{1} \preceq_{\mathcal{H}^{2}} z_{2} \Leftrightarrow\left\{\begin{array}{llll}
0 \leq x_{1} \leq x_{2} & \text { and } & 0 \leq \log \left(y_{1}\right) \leq \log \left(y_{2}\right) & \text { or } \\
x_{2} \leq x_{1} \leq 0 & \text { and } & 0 \leq \log \left(y_{1}\right) \leq \log \left(y_{2}\right) & \text { or } \\
x_{2} \leq x_{1} \leq 0 & \text { and } & \log \left(y_{2}\right) \leq \log \left(y_{1}\right) \leq 0 & \text { or } \\
0 \leq x_{1} \leq x_{2} & \text { and } & \log \left(y_{2}\right) \leq \log \left(y_{1}\right) \leq 0 &
\end{array}\right.
$$

The four conditions of this partial ordering entails that only points belonging to the same quadrant of $\mathcal{H}^{2}$ can be ordered, where the four quadrants $\left\{\mathcal{H}_{++}^{2}, \mathcal{H}_{-+}^{2}, \mathcal{H}_{--}^{2}, \mathcal{H}_{+-}^{2}\right\}$ are defined with respect to the origin $\mathcal{O}_{\mathcal{H}^{2}}=(0,1)$ which corresponds to the pure imaginary complex $z_{0}=i$. In other words, we can summarize the partial ordering (29) by saying that if $z_{1}$ and $z_{2}$ belong to the same $\mathcal{O}$-quadrant of $\mathcal{H}^{2}$ we have $z_{1} \preceq \mathcal{H}^{2} z_{2} \Leftrightarrow\left|x_{1}\right| \leq\left|x_{2}\right|$ and $\left|\log \left(x_{1}\right)\right| \leq$ $\left|\log \left(x_{2}\right)\right|$. Endowed with the partial ordering $(29), \mathcal{H}^{2}$ becomes a partially ordered set (poset) where the bottom element is $z_{0}$, but we notice that there is no top element. In addition, for any pair of point $z_{1}$ and $z_{2}$, the infimum $\curlywedge_{\mathcal{H}^{2}}$ is given by

$$
z_{1} \curlywedge_{\mathcal{H}^{2}} z_{2} \Leftrightarrow\left\{\begin{array}{cl}
\left(x_{1} \wedge x_{2}\right)+i\left(y_{1} \wedge y_{2}\right) & \text { if } z_{1}, z_{2} \in \mathcal{H}_{++}^{2} \\
\left(x_{1} \vee x_{2}\right)+i\left(y_{1} \wedge y_{2}\right) & \text { if } z_{1}, z_{2} \in \mathcal{H}_{-+}^{2} \\
\left(x_{1} \vee x_{2}\right)+i\left(y_{1} \vee y_{2}\right) & \text { if } z_{1}, z_{2} \in \mathcal{H}_{--}^{2} \\
\left(x_{1} \wedge x_{2}\right)+i\left(y_{1} \vee y_{2}\right) & \text { if } z_{1}, z_{2} \in \mathcal{H}_{+-}^{2} \\
z_{0} & \text { otherwise }
\end{array}\right.
$$

The infimum (30) extends naturally to any finite set of points in $\mathcal{H}^{2}, Z=\left\{z_{k}\right\}_{1 \leq k \leq K}$, and will be denoted by $\curlywedge_{\mathcal{H}^{2}} Z$. However, the supremum $z_{1} \curlyvee_{\mathcal{H}^{2}} z_{2}$ is not defined; or more precisely, it is defined if and only if $z_{1}$ and $z_{2}$ belong to the same quadrant, i.e., similarly to (30) mutatis mutandis $\wedge$ by $\vee$ with the "otherwise" case as "non existent". Consequently, the poset $\left(\mathcal{H}^{2}, \preceq_{\mathcal{H}^{2}}\right)$ is only a complete inf-semilattice. The fundamental property of such infimum (30) is its self-duality with respect to involution (28), i.e.,

$$
z_{1} \curlywedge_{\mathcal{H}^{2}} z_{2}=\complement\left(\complement z_{1} \curlywedge_{\mathcal{H}^{2}} \complement z_{2}\right) .
$$

Due to the strong dependency of partial ordering $\preceq_{\mathcal{H}^{2}}$ with respect to $\mathcal{O}_{\mathcal{H}^{2}}$, it is easy to see that such ordering is only invariant to transformations that does not move points from one quadrant to another one. This is the case typically for mappings as $z \mapsto \lambda \boxminus z, \lambda>0$. 


\subsection{Upper half-plane polar ordering}

Previous order $\preceq_{\mathcal{H}^{2}}$ is only a partial ordering, and consequently given any pair of points $z_{1}$ and $z_{2}$, the infimum $z_{1} \curlywedge_{\mathcal{H}^{2}} z_{2}$ can be different from $z_{1}$ and $z_{2}$. In addition, the supremum is not always defined. Let us introduce a total ordering in $\mathcal{H}$ based on hyperbolic polar coordinates, which also takes into account an ordering relationship with respect to $\mathcal{O}_{\mathcal{H}^{2}}$. Thus, given two points $\forall z_{1}, z_{2} \in \mathcal{H}$ the upper half-plane polar ordering states

$$
z_{1} \leq_{\mathcal{H}^{2}}^{\text {pol }} z_{2} \Leftrightarrow\left\{\begin{array}{l}
\eta_{1}<\eta_{2} \\
\eta_{1}=\eta_{2}
\end{array} \quad \text { or } \quad \text { and } \quad \tan \phi_{1} \leq \tan \phi_{2}\right.
$$

where $(\eta, \phi)$ are defined in Eq. (10). The polar supremum $z_{1} \vee_{\mathcal{H}^{2}}^{\text {pol }} z_{2}$ and infimum $z_{1} \wedge_{\mathcal{H}^{2}}^{\text {pol }} z_{2}$ are naturally obtained from the order (32) for any subset of points $Z$, denoted by $\bigvee_{\mathcal{H}^{2}}^{\text {pol }} Z$

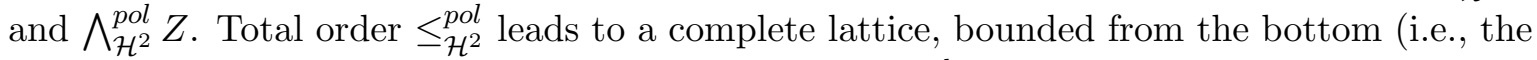
origin $\mathcal{O}_{\mathcal{H}^{2}}$ ) but not from the top. Furthermore, as $\leq_{\mathcal{H}^{2}}^{\text {pol }}$ is a total ordering, the supremum and the infimum will be either $z_{1}$ or $z_{2}$.

Polar total order is invariant to any Möbius transformation $M_{g}$ which preserves the distance to the origin (isometry group) and more generally to isotone maps in distance, i.e., $\eta\left(z_{1}\right) \leq \eta\left(z_{2}\right) \Leftrightarrow \eta\left(M_{g}\left(z_{1}\right)\right) \leq \eta\left(M_{g}\left(z_{2}\right)\right)$ but which also preserves the orientation order, i.e., order on the polar angle. This is for instance the case of orientation group $\mathrm{SO}(2)$ and the scaling maps $z \mapsto M_{g}(z)=\lambda z, 0<\lambda \in \mathbb{R}$.

We note also that instead of considering $\mathcal{O}_{\mathcal{H}^{2}}$ as the origin, the polar hyperbolic coordinates can be defined with respect to a different origin $z_{0}^{\prime}$ and consequently, the total order is adapted to the new origin (i.e., bottom element is just $z_{0}^{\prime}$ ).

One can replace in the polar ordering the distance $\operatorname{dist}_{\mathcal{H}^{2}}\left(\mathcal{O}_{\mathcal{H}^{2}}, z\right)$ by the $\alpha$-order Hellinger distance to obtain now the total ordering $\leq_{\mathcal{H}^{2}}^{\alpha-p o l}$ parametrized by $\alpha$ :

$z_{1} \leq_{\mathcal{H}^{2}}^{\alpha-\text { pol }} z_{2} \Leftrightarrow\left\{\begin{array}{l}\operatorname{dist}_{\text {Hellinger }}\left(\mathcal{O}_{\mathcal{H}^{2}}, z_{1} ; \alpha\right)<\operatorname{dist}_{\text {Hellinger }}\left(\mathcal{O}_{\mathcal{H}^{2}}, z_{2} ; \alpha\right) \\ \operatorname{dist}_{\text {Hellinger }}\left(\mathcal{O}_{\mathcal{H}^{2}}, z_{1} ; \alpha\right)=\operatorname{dist}_{\text {Hellinger }}\left(\mathcal{O}_{\mathcal{H}^{2}}, z_{2} ; \alpha\right)\end{array} \quad\right.$ or $\quad$ and $\quad \tan \phi_{1} \leq \tan \phi_{2}$

As we illustrate in Section 5, the "deformation" of the distance driven by $\alpha$ can significantly change the supremum and infimum from a set of points $Z$. Obviously, the properties of invariance of $\leq_{\mathcal{H}^{2}}^{\alpha-p o l}$ are related to the isometries of the $\alpha$-order Hellinger distance.

\subsection{Upper half-plane geodesic ordering}

As discussed above, there is a unique hyperbolic geodesic joining any pair of points. Given two points $z_{1}, z_{2} \in \mathcal{H}^{2}$ such that $x_{1} \neq x_{2}$, let $S C_{r_{1 \frown 2}}\left(a_{1 \frown 2}\right)$ be the semi-circle defining their geodesic, where the center $a_{1 \frown 2}$ and the radius $r_{1 \frown 2}$ are given by Eqs. (8). Let us denote by $z_{1 \frown 2}$ the point of $S C_{r_{1 \frown 2}}\left(a_{1 \frown 2}\right)$ having the maximal imaginary part, i.e., its imaginary part is equal to the radius: $z_{1 \frown 2}=a_{1 \frown 2}+i r_{1 \frown 2}$. 
The upper half-plane geodesic ordering $\preceq_{\mathcal{H}^{2}}^{\text {geo }}$ defines an order for points being in the same half of their geodesic semi-circle as follows,

$$
z_{1} \preceq_{\mathcal{H}^{2}}^{\text {geo }} z_{2} \Leftrightarrow\left\{\begin{array}{l}
a_{1 \frown 2} \leq x_{1}<x_{2} \\
x_{2}<x_{1} \leq a_{1} \frown 2
\end{array} \quad\right. \text { or }
$$

The property of transitivity of this partial ordering, i.e., $z_{1} \preceq_{\mathcal{H}^{2}}^{\text {geo }} z_{2}, z_{2} \preceq_{\mathcal{H}^{2}}^{\text {geo }} z_{3} \Rightarrow z_{1} \preceq_{\mathcal{H}^{2}}^{\text {geo }} z_{3}$, holds for points belonging to the same geodesic. For two points in a geodesic vertical line, $x_{1}=x_{2}$, we have $z_{1} \preceq_{\mathcal{H}^{2}}^{g e o} z_{2} \Leftrightarrow y_{2} \leq y_{1}$. We note that considering the duality with respect to the involution (28), one has

$$
z_{1} \preceq_{\mathcal{H}^{2}}^{g e o} z_{2} \Leftrightarrow \complement z_{1} \succeq_{\mathcal{H}^{2}}^{g e o} \complement z_{2} .
$$

According to this partial ordering, we define the geodesic infimum, denoted by $\curlywedge_{\mathcal{H}^{2}}^{\text {geo }}$, as the point on the geodesic joining $z_{1}$ and $z_{2}$ with maximal imaginary part, i.e., for any $z_{1}, z_{2} \in \mathcal{H}^{2}$, with $x_{1} \neq x_{2}$, we have

$$
z_{1} \curlywedge_{\mathcal{H}^{2}}^{\text {geo }} z_{2} \Leftrightarrow\left\{\begin{array}{cl}
\left(x_{1} \vee x_{2}\right)+i\left(y_{1} \vee y_{2}\right) & \text { if } x_{1}, x_{2} \leq a_{1 \frown 2} \\
\left(x_{1} \wedge x_{2}\right)+i\left(y_{1} \vee y_{2}\right) & \text { if } x_{1}, x_{2} \geq a_{1 \frown 2} \\
z_{1 \frown 2} & \text { otherwise }
\end{array}\right.
$$

If $x_{1}=x_{2}$, we have that $z_{1} \curlywedge_{\mathcal{H}^{2}}^{\text {geo }} z_{2}=x_{1}+i\left(y_{1} \vee y_{2}\right)$. In any case, we have that $\operatorname{dist}_{\mathcal{H}^{2}}\left(z_{1}, z_{2}\right)=$ $\operatorname{dist}_{\mathcal{H}^{2}}\left(z_{1}, z_{1} \curlywedge_{\mathcal{H}^{2}}^{g e o} z_{2}\right)+\operatorname{dist}_{\mathcal{H}^{2}}\left(z_{1} \curlywedge_{\mathcal{H}^{2}}^{\text {geo }} z_{2}, z_{2}\right)$. Intuitively, we notice that the geodesic infimum is the point of the geodesic farthest from the real line.

We observe that if one attempts to define the geodesic supremum from the partial ordering $\preceq_{\mathcal{H}^{2}}^{\text {geo }}$, it results that the supremum is not defined for any pair of points, i.e., supremum between $z_{1}$ and $z_{2}$ is defined if and only if both points are in the same half of its semi-circle. To tackle this limitation, we propose to define the geodesic supremum $z_{1} \curlyvee_{\mathcal{H}^{2}}^{\text {geo }} z_{2}$ by duality with respect to the involution $\complement z$, i.e.,

$$
z_{1} \curlyvee_{\mathcal{H}^{2}}^{\text {geo }} z_{2}=\complement\left(\complement z_{1} \curlywedge_{\mathcal{H}^{2}}^{\text {geo }} \complement z_{2}\right) \Leftrightarrow\left\{\begin{array}{cl}
\left(x_{1} \wedge x_{2}\right)+i\left(y_{1} \wedge y_{2}\right) & \text { if } x_{1}, x_{2} \leq a_{1 \frown 2} \\
\left(x_{1} \vee x_{2}\right)+i\left(y_{1} \wedge y_{2}\right) & \text { if } x_{1}, x_{2} \geq a_{1 \frown 2} \\
\complement z_{\complement 1 \frown \complement 2} & \text { otherwise }
\end{array}\right.
$$

where $\complement z_{\complement 1 \frown \complement 2}$ is the dual point associated to the semi-circle defined by dual points $\complement z_{1}$ and $\complement z_{2}$.

Nevertheless, in order to have a structure of complete lattice for $\left(\mathcal{H}^{2}, \preceq_{\mathcal{H}^{2}}^{\text {geo }}\right)$, it is required that the infimum and the supremum of any set of points $Z=\left\{z_{k}\right\}_{1 \leq k \leq K}$ with $K \geq 2$, are well

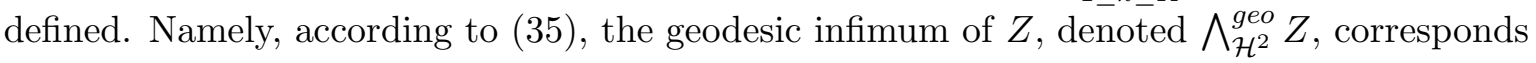
to the point $z_{\text {inf }}$ with the maximal imaginary part on all possible geodesics joining any pair of points $z_{n}, z_{m} \in Z$. In geometric terms, it means that between all these geodesics, there exists one which gives $z_{\mathrm{inf}}$. Instead of computing all the geodesics, we propose to define the infimum 


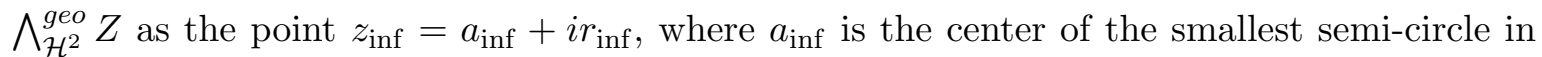
$\mathcal{H}^{2}$ of radius $r_{\text {inf }}$ which encloses all the points in the set $Z$. We have the following property

$$
\bigwedge_{\mathcal{H}^{2}}^{g e o} Z=z_{\text {inf }} \preceq_{\mathcal{H}^{2}}^{\text {geo }} z_{k}, \quad 1 \leq k \leq K,
$$

which geometrically means that the geodesic connecting $z_{\text {inf }}$ to any point $z_{k}$ of $Z$ lies always in one of the half part of the semi-circle defined by $z_{\text {inf }}$ and $z_{k}$.

In practice, the minimal enclosing semi-circle defining $z_{\text {inf }}$ can be easily computed by means of the following algorithm based on the minimum enclosing Euclidean circle $M E C$ of a set of points: (1) Working on $\mathbb{R}^{2}$, define a set of points given, on the one hand, by $Z$ and, on the other hand, by $Z^{*}$ which corresponds to the reflected points with respect to $x$-axis (complex conjugate), i.e., points $Z=\left\{\left(x_{k}, y_{k}\right)\right\}$ and points $Z^{*}=\left\{\left(x_{k},-y_{k}\right)\right\}, 1 \leq k \leq K ;(2)$ Compute the $M E C\left(Z \cup Z^{*}\right) \mapsto C_{r}(c)$, in such a way that, by a symmetric point configuration, we necessarily have the center on $x$-axis, i.e., $c=\left(x_{c}, 0\right)$; (3) The infimum $\bigwedge_{\mathcal{H}^{2}}^{g o o} Z=z_{\text {inf }}$ is given by $z_{\text {inf }}=x_{c}+i r$. Fig. 7(a)-(b) gives an example of computation of the geodesic infimum from a set of points in $\mathcal{H}^{2}$.

As for the case of two points, the geodesic supremum of $Z$ is defined by duality with respect to involution (28), i.e.,

$$
z_{\text {sup }}=\bigvee_{\mathcal{H}^{2}}^{\text {geo }} Z=\complement\left(\bigwedge_{\mathcal{H}^{2}}^{g e o} \complement Z\right)=a_{\text {sup }}+i r_{\text {sup }},
$$

with $a_{\text {sup }}=-x_{c}^{\text {dual }}$ and $r_{\text {sup }}=1 / r^{d u a l}$, where $S C_{r_{\text {dual }}}\left(x_{c}^{\text {dual }}\right)$ is the minimal enclosing semicircle from dual set of points $C Z$. An example of computing the geodesic supremum $z_{\text {sup }}$ is also given in Fig. 7(a)-(b). It is easy to see that geodesic infimum and supremum have the following properties for any $Z \subset \mathcal{H}^{2}$ :

1. $z_{\inf } \preceq_{\mathcal{H}^{2}}^{g e o} z_{\text {sup }}$;

2. $\Im\left(z_{\text {inf }}\right) \geq \Im\left(z_{k}\right)$ and $\Im\left(z_{\text {sup }}\right) \leq \Im\left(z_{k}\right), \forall z_{k} \in Z$;

3. $\bigvee_{1 \leq k \leq K} \Re\left(z_{k}\right)<\left\{\Re\left(z_{\text {inf }}\right), \Re\left(z_{\text {sup }}\right)\right\}<\bigwedge_{1 \leq k \leq K} \Re\left(z_{k}\right)$.

The proofs are straightforward from the notion of minimal enclosing semi-circle and the fact that $z_{\text {sup }}$ lies inside the semi-circle defined by $z_{\text {inf }}$.

Geodesic infimum and supremum being defined by minimal enclosing semi-circles, their invariance properties are related to translation and scaling of points in set $Z$ as defined in Section 2.4, but not to inversion. This invariance domain just corresponds to the Guts group of transformations, i.e.,

$$
\bigwedge_{\mathcal{H}^{2}}^{\text {geo }}\left\{T\left(z_{k}\right)\right\}_{1 \leq k \leq K}=T\left(\bigwedge_{\mathcal{H}^{2}}^{\text {geo }}\left\{z_{k}\right\}_{1 \leq k \leq K}\right) \text {. }
$$


As we discussed in Section 3, we do not have an explicit algorithm to compute the BurbeaRao $\alpha$-order entropy geodesic and consequently, our framework based on computing the minimum enclosing geodesic to define the infimum cannot be extended to this general case. We can nevertheless consider the example depicted in Fig. 8, where we have computed such smallest Burbea-Rao $\alpha$-order geodesic enclosing the set of points $Z$. Indeed, the example is useful to identify the limit cases with respect to $\alpha$. In fact, we note that if $\alpha \rightarrow 0$, the corresponding $\alpha$-geodesic infimum will correspond to the $z_{k}$ having the largest imaginary part, and dually for the supremum, i.e., $z_{k}$ having the smallest imaginary part. In the case of large $\alpha$, we note that the real part of both, the $\alpha$-geodesic infimum and supremum equals $\left(\vee_{1 \leq k \leq K} \Re\left(z_{k}\right)-\wedge_{1 \leq k \leq K} \Re\left(z_{k}\right)\right) / 2$, and the imaginary part of the infimum goes to $+\infty$ and of the supremum to 0 when $\alpha \rightarrow+\infty$.

\subsection{Upper half-plane asymmetric geodesic infimum/supremum}

According to the properties of geodesic infimum $z_{\text {inf }}$ and supremum $z_{\text {sup }}$ discussed above, we note that their real parts $\Re\left(z_{\text {inf }}\right)$ and $\Re\left(z_{\text {sup }}\right)$ belong to the interval bounded by the real parts of points of set $Z$. Moreover, $\Re\left(z_{\text {inf }}\right)$ and $\Re\left(z_{\text {sup }}\right)$ are not ordered between them. Therefore, the real part of supremum can be smaller than that of the infimum one. For instance, in the extreme case of a set $Z$ where all the imaginary parts are equal, the real part of its geodesic infimum and supremum are both equal to the average of the real parts of points, i.e., given $Z=\left\{z_{k}\right\}_{1 \leq k \leq K}$, if $y_{k}=y, 1 \leq k \leq K$, then $\Re\left(z_{\text {inf }}\right)=\Re\left(z_{\text {sup }}\right)=1 / K \sum_{k=1}^{K} x_{k}$. From the viewpoint of morphological image filtering, it can be potentially interesting to impose an asymmetric behavior for the infimum and supremum such that $\Re\left(z_{\text {inf }}^{-\rightarrow}\right) \leq z_{k} \leq \Re\left(z_{\text {sup }}^{-\rightarrow}\right)$, $1 \leq k \leq K$. Note that the proposed notation $-\rightarrow+$ indicates a partially ordered set on $\mathrm{x}$-axis. In order to fulfil these requirements, we can geometrically consider the rectangle bounding the minimal enclosing semi-circle, which is just of dimensions $2 r_{\text {inf }} \times r_{\text {inf }}$, and use it to define the asymmetric infimum $z_{\text {inf }}^{-\rightarrow+}$ as the upper-left corner of the rectangle. The asymmetric supremum $z_{\text {sup }}^{-\rightarrow+}$ is similarly defined from the bounding rectangle of the dual minimal enclosing semi-circle. Mathematically, given the geodesic infimum $z_{\text {inf }}$ and supremum $z_{\text {sup }}$, we have the following definitions for the asymmetric geodesic infimum and supremum:

$$
\left\{\begin{array}{c}
z_{\mathrm{inf}}^{-\rightarrow+}=\bigvee_{\mathcal{H}^{2}}^{-\rightarrow+} Z=\left(a_{\mathrm{inf}}-r_{\mathrm{inf}}\right)+i r_{\mathrm{inf}} \\
z_{\mathrm{sup}}^{-\rightarrow+}=\bigwedge_{\mathcal{H}^{2}}^{-\rightarrow} Z=-\left(x_{c}^{\text {dual }}-r^{\text {dual }}\right)+i \frac{1}{r^{d u a l}}
\end{array}\right.
$$

Remark. Geodesic infimum and supremum of Gaussian distributions. Let us consider their interpretation as infimum and supremum of a set of univariate Gaussian pdfs, see example depicted in Fig. 7. Given a set of $K$ Gaussian pdfs $N_{k}\left(\mu=\sqrt{2} x_{k}, \sigma^{2}=y_{k}^{2}\right)$, $1 \leq k \leq K$, we observe that the Gaussian pdf associated to the geodesic infimum $N_{\text {inf }}(\mu=$ $\sqrt{2} x_{\text {inf }}, \sigma^{2}=y_{\text {inf }}^{2}$ ) has a variance larger than any Gaussian of the set and its mean is a kind of barycenter between the Gaussian pdfs having a larger variance. The supremum 
Gaussian pdf $N_{\text {sup }}\left(\mu=\sqrt{2} x_{\text {sup }}, \sigma^{2}=y_{\text {sup }}^{2}\right)$ has a smaller variance than the $K$ Gaussian pdfs and its mean is between the ones of small variance. In terms of the corresponding cumulative distribution functions, we observe that geodesic supremum/infimum do not have a natural interpretation. In the case of the asymmetric Gaussian geodesic infimum $N_{\text {inf }}^{-\rightarrow+}(\mu=$ $\left.\sqrt{2} x_{\mathrm{inf}}^{-\overrightarrow{+}}, \sigma^{2}=\left(y_{\mathrm{inf}}^{-\overrightarrow{+}}\right)^{2}\right)$ and Gaussian supremum $N_{\text {sup }}^{-\overrightarrow{+}}\left(\mu=\sqrt{2} x_{\text {sup }}^{-\overrightarrow{+}}, \sigma^{2}=\left(y_{\text {sup }}^{-\overrightarrow{+}}\right)^{2}\right)$, we observe how the means are ordered with respect to the $K$ others, which also involves that the corresponding cdfs are ordered. The latter is related to the notion of stochastic dominance [30] and will be explored in detail in ongoing research. 


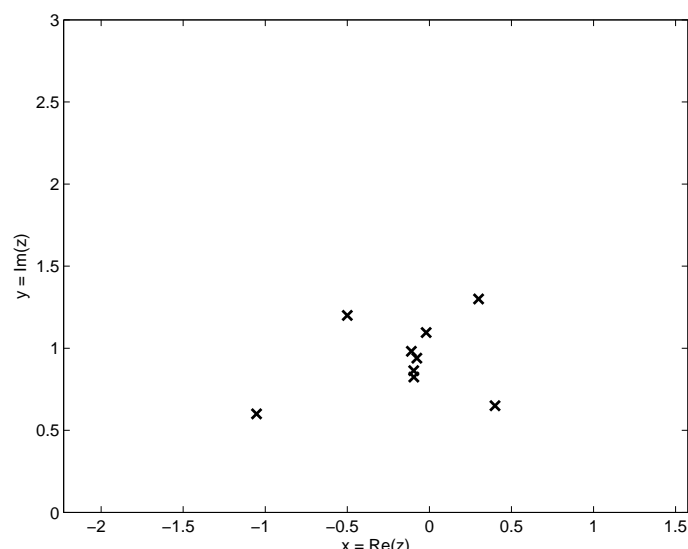

(a)

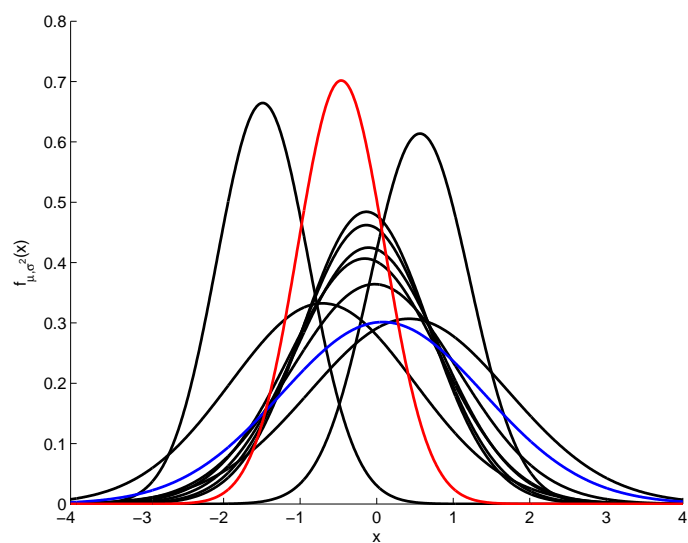

(c)

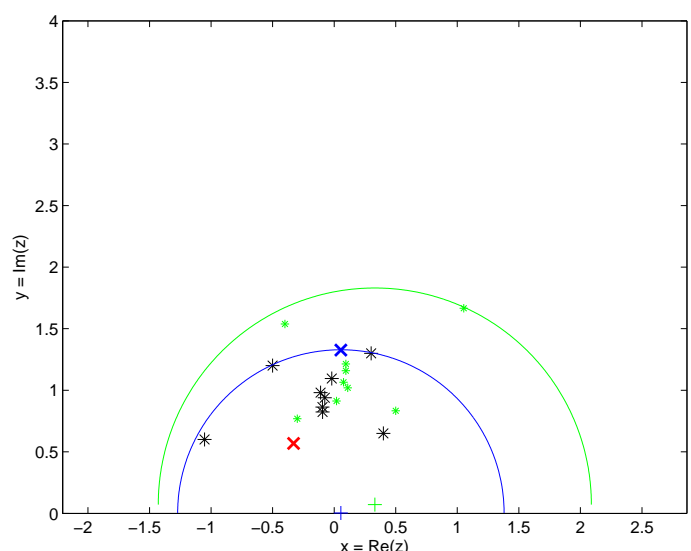

(b)

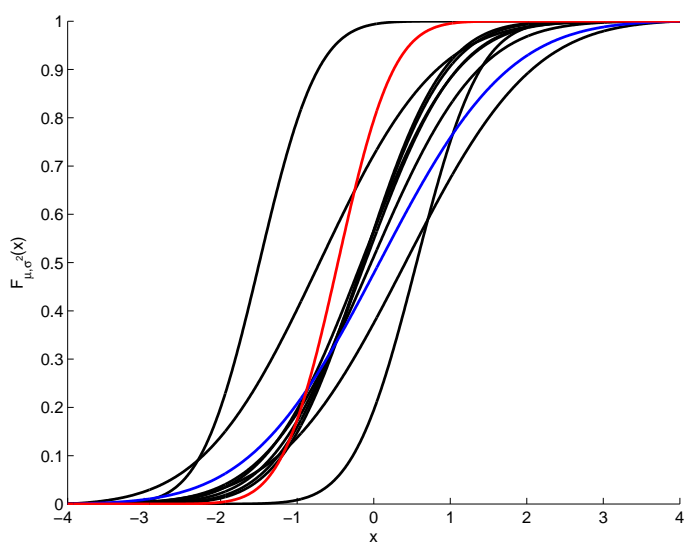

(d)

Figure 7: (a) Set of nine points in $\mathcal{H}^{2}, Z=\left\{z_{k}\right\}_{1 \leq k \leq 9}$. (b) Computation of infimum $\bigwedge_{\mathcal{H}^{2}}^{\text {geo }} Z=$ $z_{\text {inf }}$ (blue " $\times$ ") and supremum $\bigvee_{\mathcal{H}^{2}}^{\text {geo }} Z=z_{\text {sup }}$ (red " $\times$ "). Black "*" are the original points and green " $*$ " the corresponding dual ones. (c) In black, set of Gaussian pdfs associated to $Z$, i.e., $N_{k}\left(\mu=\sqrt{2} x_{k}, \sigma^{2}=y_{k}^{2}\right)$; in blue, infimum Gaussian pdf $N_{\text {inf }}\left(\mu=\sqrt{2} x_{\text {inf }}, \sigma^{2}=y_{\text {inf }}^{2}\right)$; in red, supremum Gaussian pdf $N_{\text {sup }}\left(\mu=\sqrt{2} x_{\text {sup }}, \sigma^{2}=y_{\text {sup }}^{2}\right)$. (d) Cumulative distribution functions of Gaussian pdfs from (c). 


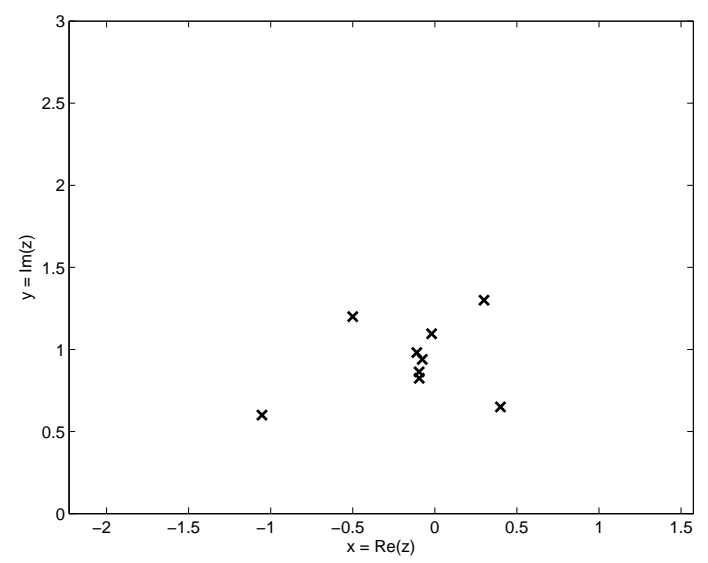

(a)

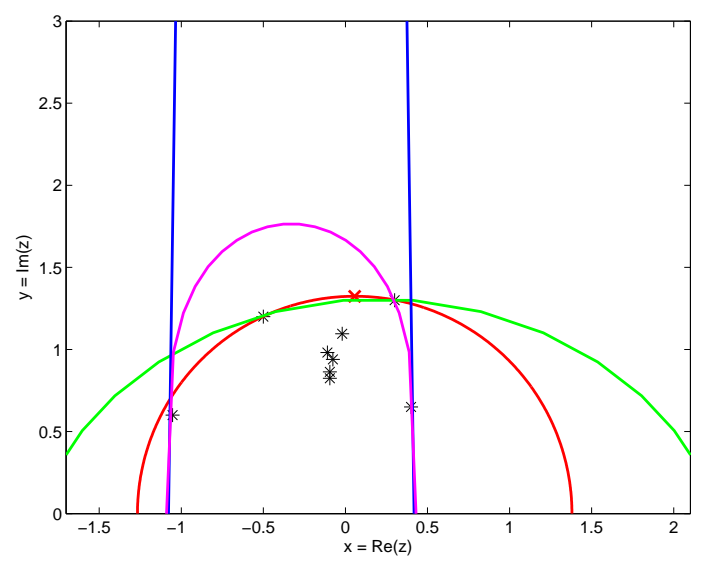

(b)

Figure 8: (a) Set of nine points in $\mathcal{H}^{2}, Z=\left\{z_{k}\right\}_{1 \leq k \leq 9}$. (b) Computation of the smallest Burbea-Rao $\alpha$-order geodesic enclosing the set $Z$, for $\alpha=0.01$ (in green), $\alpha=1$ (in red), $\alpha=5$ (in magenta), $\alpha=20$ (in blue).

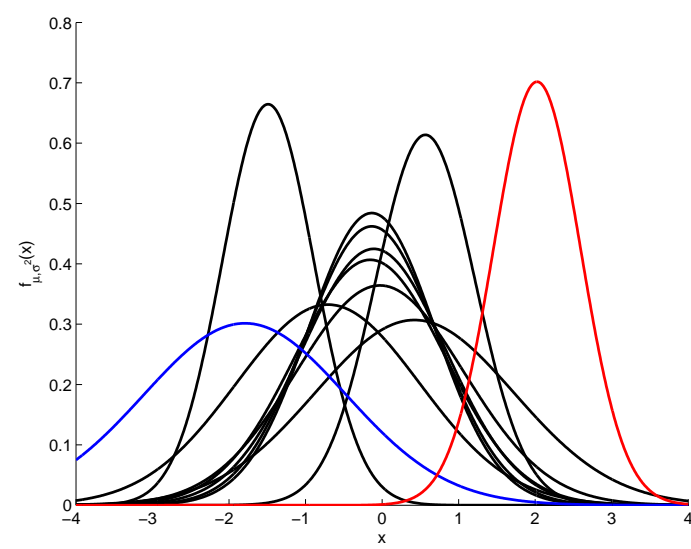

(a)

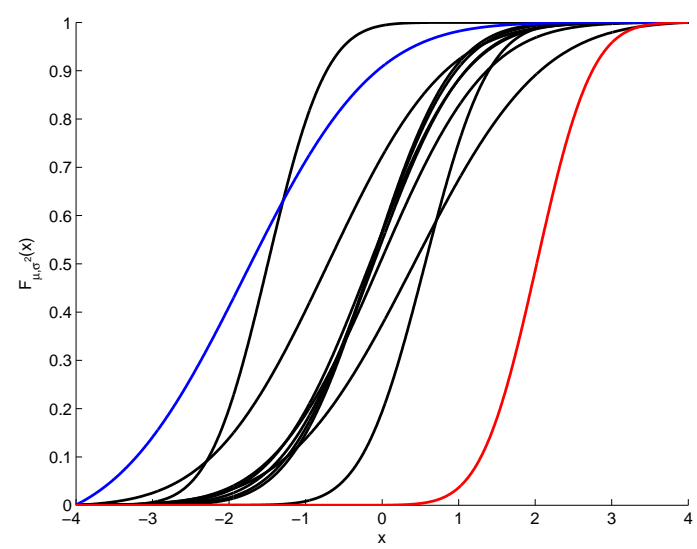

(b)

Figure 9: (a) Infimum and supremum Gaussian pdfs (in green and red respectively) from asymmetric geodesic infimum $z_{\text {inf }}^{-\rightarrow+}$ and $z_{\text {sup }}^{-\rightarrow+}$ from set of Fig. 7. (b) Cumulative distribution functions of Gaussian pdfs from (a). 


\section{Morphological operators on $\mathcal{F}\left(\Omega, \mathcal{H}^{2}\right)$ for processing univari- ate Gaussian distribution-valued images}

Let consider that $\mathcal{H}^{2}$ has been endowed with one of the partial orderings discussed above, generally denoted by $\leq$. Hence $\left(\mathcal{H}^{2}, \leq\right)$ is a poset, which has also a structure of complete lattice since we consider that infimum $\Lambda$ and supremum $\bigvee$ are defined for any set of points in $\mathcal{H}^{2}$.

\subsection{Adjunction on complete lattice $\left(\mathcal{H}^{2}, \leq\right)$}

The operators $\varepsilon: \mathcal{H}^{2} \rightarrow \mathcal{H}^{2}$ and $\delta: \mathcal{H}^{2} \rightarrow \mathcal{H}^{2}$ are an erosion and a dilation if they commute respectively with the infimum and the supremum: $\varepsilon\left(\bigwedge_{k} z_{k}\right)=\bigwedge_{k} \varepsilon\left(z_{k}\right)$ and $\delta\left(\bigvee_{k} z_{k}\right)=$ $\bigvee_{k} \delta\left(z_{k}\right)$, for every set $\left\{z_{k}\right\}_{1 \leq k \leq K}$. Erosion and dilation are increasing operators, i.e., $\forall z, z^{\prime} \in$ $\mathcal{H}^{2}$, if $z \leq z^{\prime}$ then $\varepsilon(z) \leq \varepsilon\left(z^{\prime}\right)$ and $\delta(z) \leq \delta\left(z^{\prime}\right)$. Erosion and dilation are related by the notion of adjunction [29, 21], i.e.,

$$
\delta(z) \leq z^{\prime} \Leftrightarrow z \leq \varepsilon\left(z^{\prime}\right) ; \forall z, z^{\prime} \in \mathcal{H}^{2} .
$$

Adjunction law (39) is of fundamental importance in mathematical morphology since it allows to define a unique dilation $\delta$ associated to a given erosion $\varepsilon$, i.e., $\delta\left(z^{\prime}\right)=\bigwedge\left\{z \in \mathcal{H}^{2}: z^{\prime} \leq\right.$ $\varepsilon(z)\}, z^{\prime} \in \mathcal{H}^{2}$. Similarly one can define a unique erosion from a given dilation: $\varepsilon(z)=$ $\bigvee\left\{z^{\prime} \in \mathcal{H}^{2}: \delta\left(z^{\prime}\right) \leq z\right\}, z \in \mathcal{H}^{2}$. Given an adjunction $(\varepsilon, \delta)$, their composition product operators, $\gamma(z)=\delta(\varepsilon(z))$ and $\varphi(z)=\varepsilon(\delta(z))$ are respectively an opening and a closing, which are the basic morphological filters having very useful properties [29, 21]: idempotency $\gamma \gamma(z)=\gamma(z)$, anti-extensivity $\gamma(z) \leq z$ and extensivity $z \leq \varphi(z)$, and increaseness. Another relevant result is the fact, given an erosion $\varepsilon$, the opening and closing by adjunction are exclusively defined in terms of the erosion [21] as $\gamma(z)=\bigwedge\left\{z^{\prime} \in \mathcal{H}^{2}: \varepsilon(z) \leq \varepsilon\left(z^{\prime}\right)\right\}, \varphi(z)=$ $\bigwedge\left\{\varepsilon\left(z^{\prime}\right): z^{\prime} \in \mathcal{H}^{2}, z \leq \varepsilon\left(z^{\prime}\right)\right\}, \forall z \in \mathcal{H}^{2}$.

In the case of complete inf-semilattice $\left(\mathcal{H}^{2}, \leq\right)$, where infimum $\bigwedge$ is defined but supremum $\bigvee$ is not necessarily, we have the following particular results [23, 22]: (a) it is always possible to associate an opening $\gamma$ to a given erosion $\varepsilon$ by means of $\gamma(z)=\bigwedge\left\{z^{\prime} \in \mathcal{H}^{2}: \varepsilon(z) \leq \varepsilon\left(z^{\prime}\right)\right\}$, (b) even though the adjoint dilation $\delta$ is not well-defined in $\mathcal{H}^{2}$, it is always well-defined for elements on the image of $\mathcal{H}^{2}$ by $\varepsilon$, and (c) $\gamma=\delta \varepsilon$. The closing defined by $\varphi=\varepsilon \delta$ is only partially defined. Obviously, in the case of inf-semilattice, it is still possible to define $\delta$ such that $\bigvee \delta\left(z_{k}\right)=\delta \bigvee\left(z_{k}\right)$ for families for which supremum $\bigvee$ exist.

\subsection{Erosion and dilation in $\mathcal{F}\left(\Omega, \mathcal{H}^{2}\right)$}

If $\left(\mathcal{H}^{2}, \leq\right)$ is a complete lattice, the set of images $\mathcal{F}\left(\Omega, \mathcal{H}^{2}\right)$ is also a complete lattice defined as follows: for all $f, g \in \mathcal{F}\left(\Omega, \mathcal{H}^{2}\right)$, (i) $f \leq g \Leftrightarrow f(p) \leq g(p), \forall p \in \Omega$; (ii) $(f \wedge g)(p)=$ $f(p) \wedge g(p), \forall p \in \Omega$; (iii) $(f \vee g)(p)=f(p) \vee g(p), \forall p \in \Omega$, where $\wedge$ and $\vee$ are the infimum 


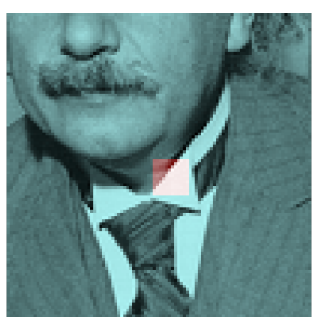

(a)

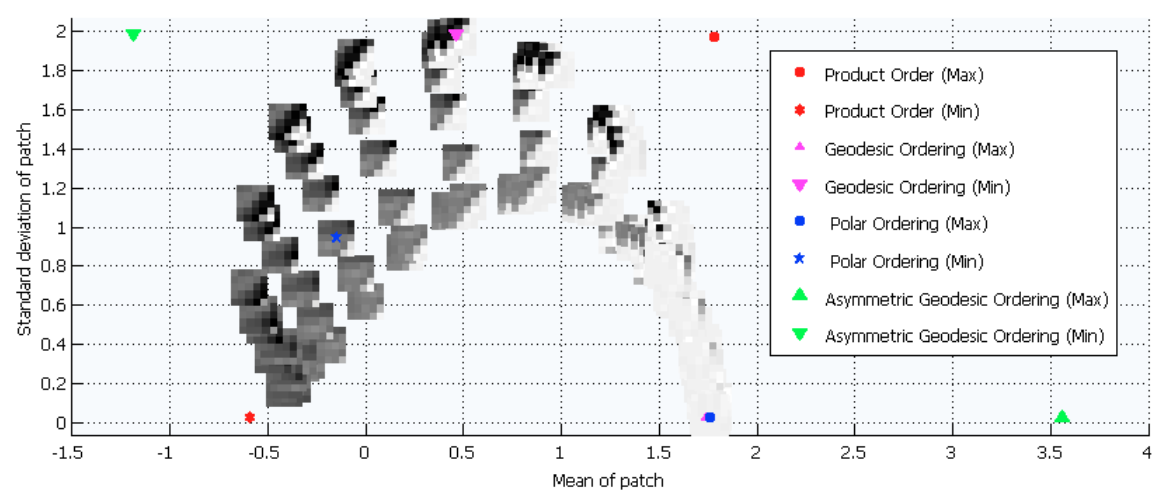

(b)

Figure 10: Supremum and infimum of a set of 25 patches parameterized by their mean and standard deviation: (a) in red the region where the overlapped patches are taken; (b) embedding into the space $\mathcal{H}^{2}$ according to the coordinates $\mu / \sqrt{2}$ and $\sigma$ and corresponding sup and inf for the different ordering strategies.

and supremum in $\mathcal{H}^{2}$. One can now define the following adjoint pair of flat erosion $\varepsilon_{B}(f)$ and flat dilation $\delta_{B}(f)$ of each pixel $p$ of image $f[29,21]$ :

$$
\begin{aligned}
& \varepsilon_{B}(f)(p)=\bigwedge_{q \in B(p)} f(p+q), \\
& \delta_{B}(f)(p)=\bigvee_{q \in B(p)} f(p-q),
\end{aligned}
$$

such that

$$
\delta_{B}(f)(p) \leq g(p) \Leftrightarrow f(p) \leq \varepsilon_{B}(g)(p) ; \forall f, g \in \mathcal{F}\left(\Omega, \mathcal{H}^{2}\right) .
$$

where set $B$ is called the structuring element, which defines the set of points in $\Omega$ when it is centered at point $p$, denoted $B(p)[31]$. These operators, which are translation invariant, can be seen as constant-weight (this is the reason why they are called flat) inf/sup-convolutions, where the structuring element $B$ works as a moving window.

The above erosion (resp. dilation) moves object edges within the image in such a way that it expands image structures with values in $\mathcal{H}^{2}$ close to the bottom element (resp. close to the top) of the lattice $\mathcal{F}\left(\Omega, \mathcal{H}^{2}\right)$ and shrinks objects with values close to the top element (resp. close to the bottom).

Let us consider now the various cases of supremum and infimum introduced above. In order to support the discussion, we have included an example in Fig. 10. In fact, we have 
taken all the patches of size $5 \times 5$ pixels surrounding one of the pixels from image of Fig. 1 . The 25 patches are then embedded into the space $\mathcal{H}^{2}$ according to the coordinates $\mu / \sqrt{2}$ and $\sigma$. Finally, the supremum and infimum of this set of points are computed for the different cases. It just corresponds to the way to obtain respectively the dilation and erosion for the current pixel center of the red region in image Fig. 10(a).

Everything works perfectly for the supremum and infimum in the upper half-plane product ordering $\bigvee_{\mathcal{H}^{2}}$ and $\bigwedge_{\mathcal{H}^{2}}$, which consequently can be used to construct dilation and erosion operators in $\mathcal{F}\left(\Omega, \mathcal{H}^{2}\right)$. In fact, this is exactly equivalent to the classical operators applied on the real and imaginary parts separately.

Similarly, the ones for the upper half-plane polar ordering $\bigvee_{\mathcal{H}^{2}}^{\text {pol }}$ and $\bigwedge_{\mathcal{H}^{2}}^{\text {pol }}$, based on a total partial ordering, also lead respectively to dilation and erosion operators. The erosion produces a point which corresponds here to the patch closer to the origin. That means a patch of intermediate mean and standard deviation intensity since the image intensity is normalized, see Section 5.4. On the contrary, the dilation gives a point associated to the farthest patch from the origin. In this example, an homogenous bright patch. Note that patches of great distance correspond to the most "contrasted" ones on the image: either homogeneous patches of dark or bright intensity or patches with a strong variation in intensity (edge patches).

We note that for the symmetric ordering $\preceq_{\mathcal{H}^{2}}$ one only has an inf-semilattice structure associated to ${\widehat{\mathcal{H}^{2}}}$. However, in the case of the upper half-plane geodesic ordering, the pair of operators (40) and (41) associated to our supremum $\bigvee_{\mathcal{H}^{2}}^{g e o}$ and infimum $\bigwedge_{\mathcal{H}^{2}}^{\text {geo }}$ will not verify the adjunction (42). Same limitation also holds for the upper half-plane asymmetric geodesic supremum and infimum. Hence, the geodesic supremum and infimum do not strictly involve a pair of dilation and erosion in the mathematical morphology sense. Nevertheless, we can compute both operators and use them to filter out images in $\mathcal{F}\left(\Omega, \mathcal{H}^{2}\right)$ without problem. From the example of Fig. 10 we observe that the geodesic infimum gives a point with a standard deviation equal or larger than any of the patches and a mean intermediate between the patches of high standard deviation. The supremum involves a point of standard deviation smaller than (or equal to) the others, and the mean is obtained by averaging around the mean of the ones with a small standard deviation. Consequently the erosion involves a nonlinear filtering which enhances the image zones of high standard deviation, typically the contours. The dilation enhances the homogenous zones. The asymmetrization produces operators where the dilation and erosion have the same interpretation for the mean as the classical ones but the filtering effects are driven by the zones of low or high standard deviation.

\subsection{Opening and closing in $\mathcal{F}\left(\Omega, \mathcal{H}^{2}\right)$}

Given the adjoint image operators $\left(\varepsilon_{B}, \delta_{B}\right)$, the opening and closing by adjunction of image $f$, according to structuring element $B$, are defined as the composition operators $[29,21]$ :

$$
\begin{aligned}
\gamma_{B}(f) & =\delta_{B}\left(\varepsilon_{B}(f)\right), \\
\varphi_{B}(f) & =\varepsilon_{B}\left(\delta_{B}(f)\right) .
\end{aligned}
$$


Openings and closings are referred to as morphological filters, which remove objects of image $f$ that do not comply with a criterion related, on the one hand, to the invariance of the object support to the structuring element $B$ and, on the other hand, to the values of the object on $\mathcal{H}^{2}$ which are far from (in the case of the opening) or near to (in the case of the closing) the bottom element of $\mathcal{H}^{2}$ according to the given partial ordering $\leq$.

Once the pairs of dual operators $\left(\varepsilon_{B}, \delta_{B}\right)$ and $\left(\gamma_{B}, \varphi_{B}\right)$ are defined, the other morphological filters and transformation can be naturally defined [31] for images in $\mathcal{F}\left(\Omega, \mathcal{H}^{2}\right)$. We limit here the illustrative examples to the basic ones.

Following our analysis on the particular cases of ordering and supremum/infimum in $\mathcal{H}^{2}$, we can conclude that opening and closing in $\mathcal{F}\left(\Omega, \mathcal{H}^{2}\right)$ are well formulated for the upper half-plane product ordering and the upper half-plane polar ordering. In the case of the upper half-plane symmetric ordering, the opening is always defined and the closing cannot be computed. Again, we should insist on the fact that for the upper half-plane geodesic ordering, the composition operators obtained by supremum $\bigvee_{\mathcal{H}^{2}}^{\text {geo }}$ and infimum $\bigwedge_{\mathcal{H}^{2}}^{\text {geo will not }}$ produce opening and closing stricto sensu. Notwithstanding, the corresponding composition operators yield a regularization effect of $\mathcal{F}\left(\Omega, \mathcal{H}^{2}\right)$-images which can be of interest for practical applications.

\subsection{Application to morphological processing univariate Gaussian distribu- tion valued images}

Example 1. A first example of morphological processing for images in $\mathcal{F}\left(\Omega, \mathcal{H}^{2}\right)$ is given in Fig. 11-12. The starting point is a standard gray-level image $g \in \mathcal{F}(\Omega, \mathbb{R})$, which is mapped to the image $f(p)=f_{x}(p)+i f_{y}(p)$ by the following transformations: (1) the image is normalized to have zero mean and a unit variance; $(2)$ the real and imaginary components of $f(p)$ are obtained by computing respectively the mean and standard deviation over a patch centered at $p$ of radius $W$ pixels (in the example $W=4$ ); i.e.,

$$
g(p) \mapsto \hat{g}(p)=\frac{g(p)-\operatorname{Mean}(g)}{\sqrt{\operatorname{Var}(g)}} \mapsto f(p)=\operatorname{Mean}_{W}(\hat{g})(p)+i \sqrt{\operatorname{Var}_{W}(\hat{g})(p)} .
$$

We note that definition of our representation space $f_{y}(p)>0$. It means that the variance of each patch should always be bigger than zero and obviously this is not the case in constant patches. In order to cope with this problem, we propose to add a $\epsilon$ to the value of the standard deviation.

Fig. 11-12 gives a comparison of morphological erosions $\varepsilon_{B}(f)(p)$ and openings $\gamma_{B}(f)(p)$ on this image $f$ using the five complete (inf-semi)lattice of $\mathcal{H}^{2}$ considered in the paper. We have included also the pseudo-erosions and pseudo-openings associated to the geodesic supremum and infimum and the asymmetric geodesic ones. The same structuring element $B$, a square of $5 \times 5$ pixels, has been used for all the examples. First of all, we remind 
again that working on the product complete lattice $\left(\mathcal{H}^{2}, \leq_{\mathcal{H}^{2}}\right)$ is equivalent to a marginal processing of real and imaginary components. As expected, the symmetric ordering-based inf-semilattice $\left(\mathcal{H}^{2}, \preceq_{\mathcal{H}^{2}}\right)$ and polar ordering-based lattice $\left(\mathcal{H}^{2}, \leq_{\mathcal{H}^{2}}^{\text {pol }}\right)$ produce rather similar results for openings. We observe that in both cases the opening produces a symmetric filtering effect between bright/dark intensity in the mean and standard deviation component. But it is important to remark that the processing effects depend on how image components are valued with respect to the origin $z_{0}=(0,1)$. This is the reason why it is proposed to always normalize by mean/variance the image.

The results of the pseudo-openings produced by working on geodesic lattice $\left(\mathcal{H}^{2}, \bigvee_{\mathcal{H}^{2}}^{\text {geo }}, \bigwedge_{\mathcal{H}^{2}}^{\text {geo }}\right)$ and asymmetric geodesic lattice $\left(\mathcal{H}^{2}, \bigwedge_{\mathcal{H}^{2}}^{-+}, \bigvee_{\mathcal{H}^{2}}^{-+}\right)$involves a processing which is mainly driven by the values of the standard deviation. Hence, the filtering effects are potentially more interesting for applications requiring to deal with pixel uncertainty, either in a symmetric processing of both bright/dark mean values with $\left(\mathcal{H}^{2}, \bigvee_{\mathcal{H}^{2}}^{g e o}, \bigwedge_{\mathcal{H}^{2}}^{\text {geo }}\right)$ or in a more classical morphological asymmetrization with $\left(\mathcal{H}^{2}, \bigwedge_{\mathcal{H}^{2}}^{-\rightarrow}, \bigvee_{\mathcal{H}^{2}}^{-\rightarrow+}\right)$.

Example 2. Fig. 13 illustrates a comparative example of erosions $\varepsilon_{B}(f)(p)$ on a very noisy image $g(p)$. We note that $g(p)$ is mean centered. The "noise" is related to an acquisition at the limit of exposure time/spatial resolution. We consider an image model $f(p)=f_{x}(p)+i f_{y}(p)$, where $f_{x}(p)=g(x)$ and $f_{y}(p)$ is the standard deviation of intensities in a patch of radius equal to 4 pixels. In fact, the results of erosion obtained by the product and symmetric partial orderings are compared to the ones obtained by polar ordering and more generally by the $\alpha$-polar ordering with four values of $\alpha$. We observe, on the one hand, polar orderings are more relevant than the product or symmetric ones. As expected, the $\alpha$-polar erosion with $\alpha=1$ is almost equivalent to the hyperbolic polar ordering. We note, on the other hand, the interest of the limit cases of $\alpha$-polar erosion. The erosion for small $\alpha$ produces a strongly regularized image where the bright/dark objects with respect to the background has been nicely enhanced. In the case of large $\alpha$, the background (i.e., pixels values close to the origin in $\mathcal{H}^{2}$ ) is enhanced, which involves removing all the image structures smaller than the structuring element $B$.

Example 3. In Fig. 14 a limited comparison for the case of dilation $\delta_{B}(f)(p)$ is depicted. The image $f(p)=f_{x}(p)+i f_{y}(p)$ is obtained similarly to the case of Example 1. We can compare the supremum by product ordering with those obtained by the polar supremum and the $\alpha$-polar supremum, with $\alpha=0.01$. The analysis is similar to the previous case.

Example 4. Fig. 15 involves again the noisy retinal image, and it shows a comparison of results from (pseudo-)opening $\gamma_{B}(f)(p)$ and (pseudo-)closing $\varphi_{B}(f)(p)$ obtained for the product ordering, the geodesic lattice $\left(\mathcal{H}^{2}, \bigvee_{\mathcal{H}^{2}}^{\text {geo }}, \bigwedge_{\mathcal{H}^{2}}^{\text {geo }}\right)$ and the asymmetric geodesic lattice $\left(\mathcal{H}^{2}, \bigwedge_{\mathcal{H}^{2}}^{-\rightarrow}, \bigvee_{\mathcal{H}^{2}}^{-\rightarrow}\right)$. The structuring element $B$ is a square of $5 \times 5$ pixels. In order to be able to compare their enhancement effects with an averaging operator, it is also given the result of filtering by computing the minimax center in a square of $5 \times 5$ pixels $[8,2]$, see Remark in Section 2.5. We note that operators associated to the asymmetric geodesic supremum and infimum yield mean images relatively similar to the standard ones underlaying 
the supremum and infimum in the product lattice. However, including the information given by the local standard deviation, the contrast of the structures is better in the asymmetric geodesic supremum and infimum. Nevertheless, we observe that the operators by geodesic supremum and infimum also produce in this example a significant regularization of the image. By the way, we note that the corresponding geodesic pseudo-opening and pseudo-closing give rather similar mean images but different standard deviation images, as expected by the formulation of the geodesic supremum and infimum.

Example 5. The example given in Fig. 16 corresponds to an image $f(p)=f_{x}(p)+i f_{y}(p)$ obtained by multiple acquisition of a sequence of 100 frames, where $f_{x}(p)$ represents the mean intensity at each pixel and $f_{y}(p)$ the standard deviation of intensity along the sequence. The 100 frames have been taken from a stationary camera.

The goal of the example is to show how to extract image objects of large intensity and support size smaller than the structuring element (here a square of $7 \times 7$ pixels) using the residue between the original image $f(p)$ and its filtered image by opening $\gamma_{B}(f)$. In the case of images on $\mathcal{F}\left(\Omega, \mathcal{H}^{2}\right)$, the residue is defined as the pixelwise hyperbolic distance between them. In this case study, results on processing on polar ordering-based lattice versus asymmetric geodesic lattice are compared.

\subsection{Conclusions on morphological operators for $\mathcal{F}\left(\Omega, \mathcal{H}^{2}\right)$ images}

Based on the discussion given in Section 5.2 as well as on the examples from Section 5.4, we can draw some conclusions on the experimental part of this chapter.

- First of all, we note that the examples considered here are only a preliminary exploration on the potential applications of morphological processing univariate Gaussian distribution-valued images.

- We have two main case studies. First, standard images which are embedded into the Poincaré upper-half plane representation by parameterization of each local patch by its mean and standard deviation. Second, images which naturally involves a distribution of values at each pixel. Note that in the first case, the information of standard deviation is mainly associated to discriminate between homogenous zones and inhomogeneous ones (textures or contours). In the second case, the standard deviation involves relevant information on the nature of the noise during the acquisition.

- For any of these two cases, we should remark that different alternatives of ordering and derived operators considered in the paper will produce nonlinear processing: its main property is that filtering effects are strongly driven by the standard deviation.

- Upper half-plane product ordering is nothing more than standard processing of mean and standard deviation separately. The symmetric ordering leading to an inf-semilattice has a limited interest since similar effects are obtained by the polar ordering. 
- Upper half-plane polar ordering using standard hyperbolic polar coordinates or the $\alpha$ order Hellinger distance produces morphological operators appropriate for image regularization and enhancement. We remind that points close to the origin (selected by the erosion) correspond in the case of the patches to those of intermediate mean and standard deviation intensity after normalization. On the contrary, patches far from the origin correspond to the "contrasted" ones: either homogeneous patches of dark or bright intensity or patches with a strong variation in intensity (edge patches).

We note that with respect to filters based on averaging, the half-plane polar dilation/erosion as well as their product operators, produces strong simplified images where the edges and the main objects are enhanced without any blurring effect.

From our viewpoint this is useful for both cases of images. Then, the choice of a high or low value for $\alpha$ will depend on the particular nature of the features to be enhanced. In any case, this parameter can be optimized.

- Upper half-plane geodesic ordering involves a nonlinear filtering framework which takes into account the intrinsic geometry of $\mathcal{H}^{2}$. It is mainly based on the notion of minimal enclosing geodesic which covers the set of points.

In practice, the geodesic infimum gives a point with a standard deviation equal to or larger than any of the point and a mean which can be seen as intermediate between the mean values of high standard deviation. The supremum produces a point of standard deviation equal to or smaller than the others, and the mean is obtained by averaging around the mean of the ones having a small standard deviation.

Consequently the erosion involves a nonlinear filtering which enhances the image zones of high standard deviation, typically the contours. The dilation enhances the homogenous zones. We should note that the processed mean images by the composition of these two operators (i.e., openings and closings) are strongly enhanced by increasing their bright/dark contrast. Therefore, it should be considered as an appropriate tool for contrast structure enhancement on irregular backgrounds.

The asymmetric version of the geodesic ordering involves that dilation and erosion have the same interpretation for the mean as the classical ones but the filtering effects are driven by the zones of low or high standard deviation. These operators are potentially useful for object extraction by residue between the original image and the opening/closing. In comparison with classical residues, the new ones produce sharper extracted objects. 


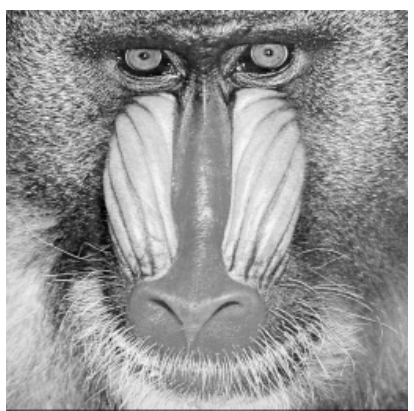

(a) $g(p)$

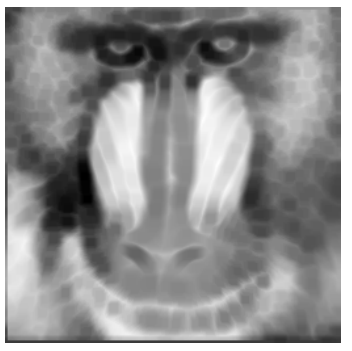

$(\mathrm{d}-1) \varepsilon_{B}(f)(p),\left(\mathcal{H}^{2}, \leq_{\mathcal{H}^{2}}\right)$
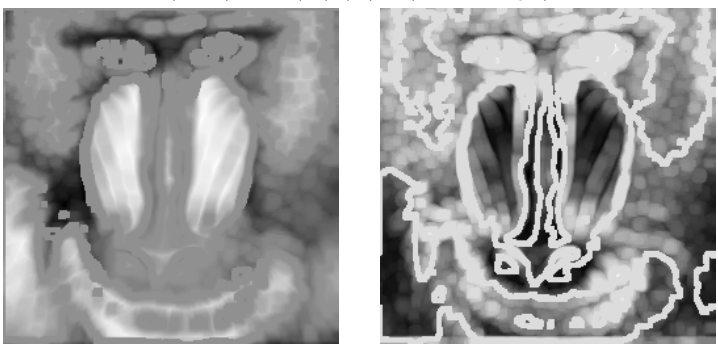

$(\mathrm{d}-2) \varepsilon_{B}(f)(p),\left(\mathcal{H}^{2}, \preceq_{\mathcal{H}^{2}}\right)$

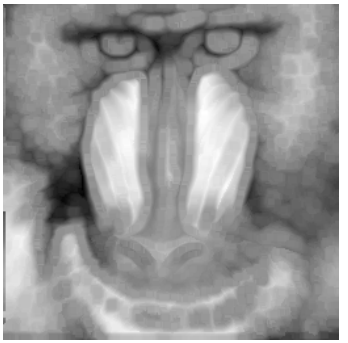

$(\mathrm{d}-3) \varepsilon_{B}(f)(p),\left(\mathcal{H}^{2}, \leq_{\mathcal{H}^{2}}^{\text {pol }}\right)$

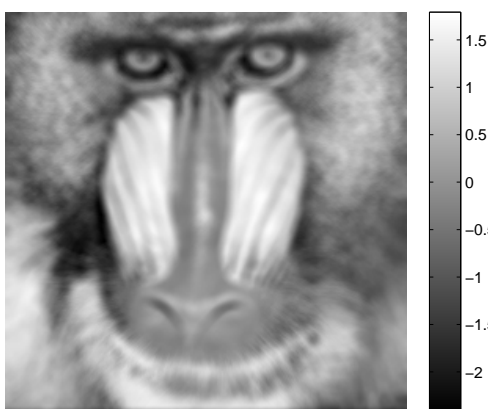

(b) $f_{x}(p)$

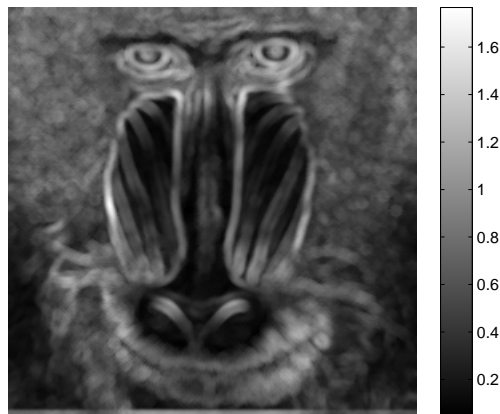

(c) $f_{y}(p)$

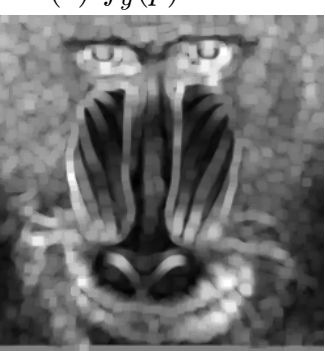

$(\mathrm{e}-1) \gamma_{B}(f)(p),\left(\mathcal{H}^{2}, \leq_{\mathcal{H}^{2}}\right)$
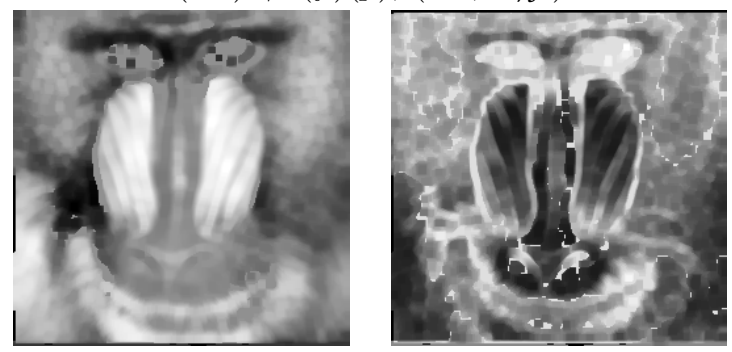

$(\mathrm{e}-2) \gamma_{B}(f)(p),\left(\mathcal{H}^{2}, \preceq \mathcal{H}^{2}\right)$
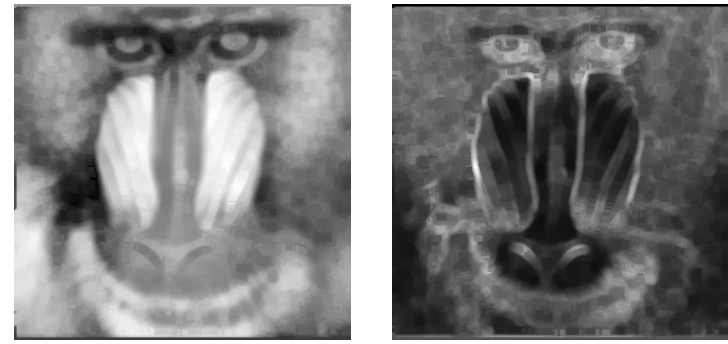

(e-3) $\gamma_{B}(f)(p),\left(\mathcal{H}^{2}, \leq_{\mathcal{H}^{2}}^{\text {pol }}\right)$

Figure 11: Comparison of morphological erosions and openings of an image $f \in \mathcal{F}\left(\Omega, \mathcal{H}^{2}\right)$ : (a) Original real-valued image $g(p) \in \mathcal{F}(\Omega, \mathbb{R})$ used to simulate (see the text) the image $f(p)=f_{x}(p)+i f_{y}(p)$, where (b) and (c) gives respectively the real and imaginary components. (d-) and (e-) depict respectively the erosion $\varepsilon_{B}(f)(p)$ and opening $\gamma_{B}(f)(p)$ of image $f(p)$ for five orderings on the upper half-plane. The structuring element $B$ is a window of $5 \times 5$ pixels. Continued in next figure. 

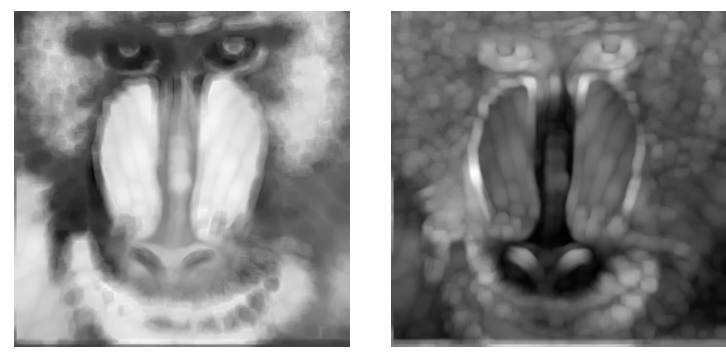

$(\mathrm{d}-4) \varepsilon_{B}(f)(p),\left(\mathcal{H}^{2}, \bigvee_{\mathcal{H}^{2}}^{g e o}, \bigwedge_{\mathcal{H}^{2}}^{g e o}\right)$

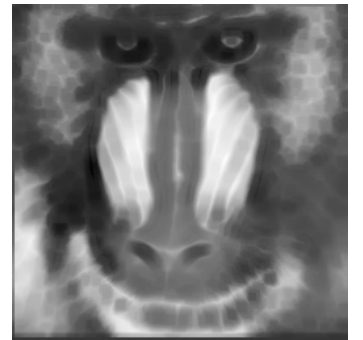

$(\mathrm{d}-5) \varepsilon_{B}(f)(p),\left(\mathcal{H}^{2}, \bigwedge_{\mathcal{H}^{2}}^{-\overrightarrow{+}}, \bigvee_{\mathcal{H}^{2}}^{-\rightarrow+}\right)$
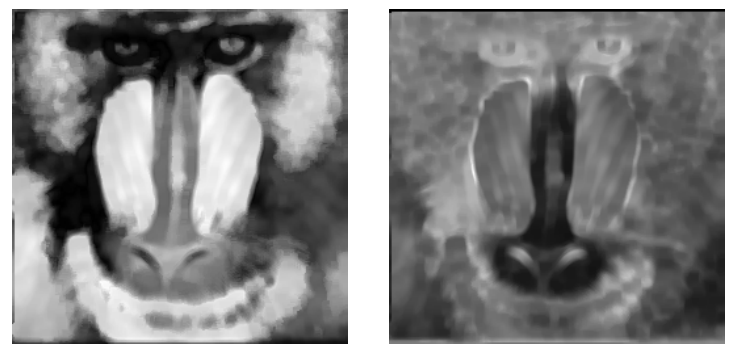

$(\mathrm{e}-4) \gamma_{B}(f)(p),\left(\mathcal{H}^{2}, \bigvee_{\mathcal{H}^{2}}^{g e o}, \bigwedge_{\mathcal{H}^{2}}^{g e o}\right)$
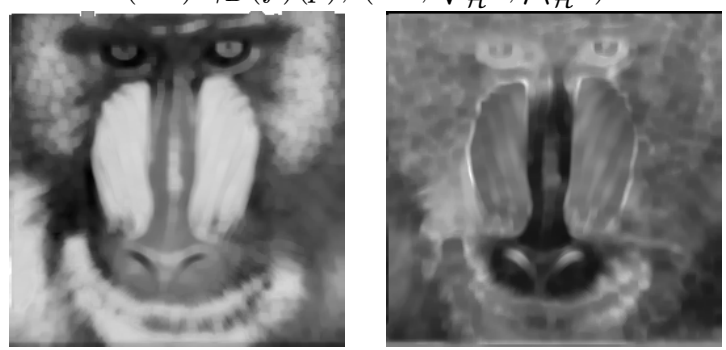

$(\mathrm{e}-5) \gamma_{B}(f)(p),\left(\mathcal{H}^{2}, \bigwedge_{\mathcal{H}^{2}}^{-\overrightarrow{+}}, \bigvee_{\mathcal{H}^{2}}^{-\rightarrow+}\right)$

Figure 12: Continuation from previous figure. 

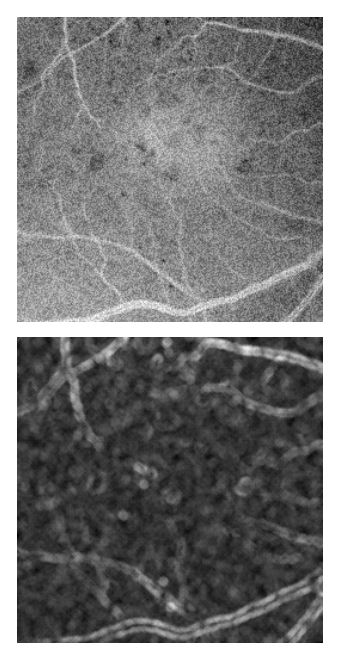

(a) $f(p)$
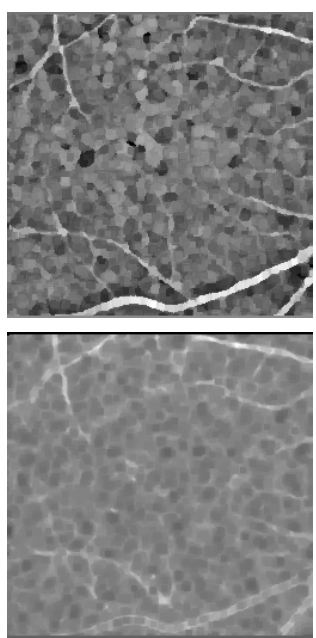

(e) $\varepsilon_{B}(f)(p)$,

$\left(\mathcal{H}^{2}, \leq_{\mathcal{H}^{2}}^{\alpha-p o l}\right), \alpha=0.01$
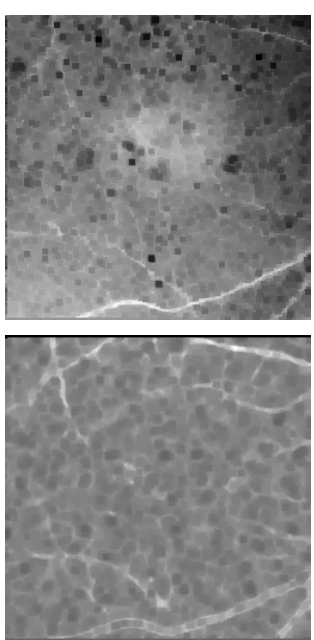

(c) $\varepsilon_{B}(f)(p)$,

$\left(\mathcal{H}^{2}, \leq_{\mathcal{H}^{2}}\right)$
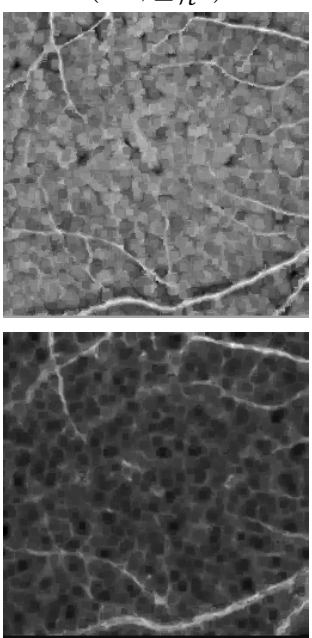

(f) $\varepsilon_{B}(f)(p)$,

$\left(\mathcal{H}^{2}, \leq_{\mathcal{H}^{2}}^{\alpha-p o l}\right), \alpha=1$
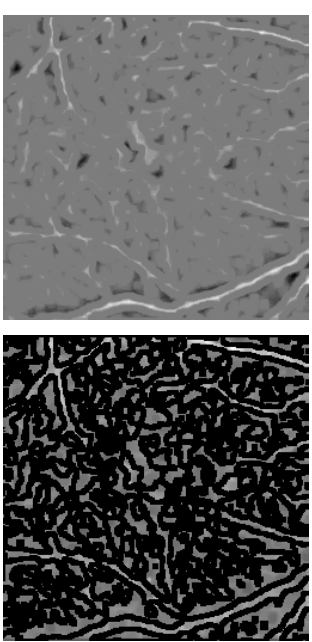

(c) $\varepsilon_{B}(f)(p)$,

$\left(\mathcal{H}^{2}, \preceq_{\mathcal{H}^{2}}\right)$
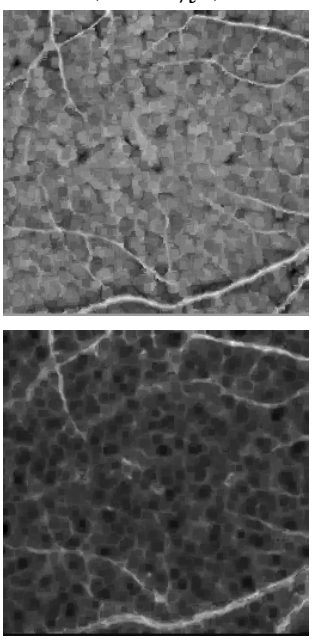

(g) $\varepsilon_{B}(f)(p)$,

$\left(\mathcal{H}^{2}, \leq_{\mathcal{H}^{2}}^{\alpha-p o l}\right), \alpha=20$
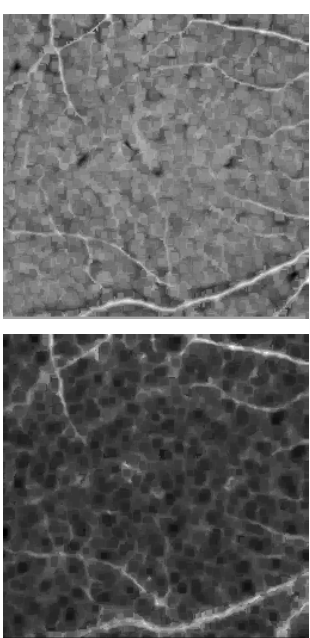

(d) $\varepsilon_{B}(f)(p)$, $\left(\mathcal{H}^{2}, \leq_{\mathcal{H}^{2}}^{\text {pol }}\right)$
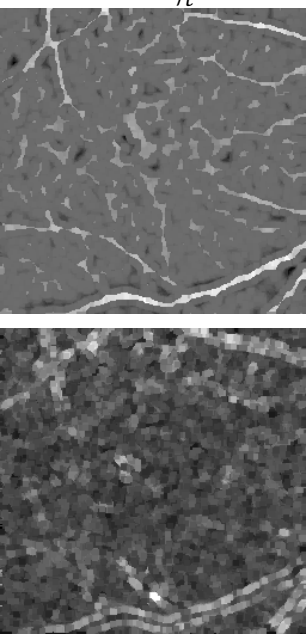

(h) $\varepsilon_{B}(f)(p)$,

$\left(\mathcal{H}^{2}, \leq_{\mathcal{H}^{2}}^{\alpha-p o l}\right), \alpha=200$

Figure 13: Comparison of erosion of Gaussian distribution-valued noisy image $\varepsilon_{B}(f)(p)$ : (a) Original image $f \in \mathcal{F}\left(\Omega, \mathcal{H}^{2}\right)$, showing both the real and the imaginary components; (b) upper half-plane product ordering (equivalent to standard processing); (c) upper half-space symmetric ordering; (d) upper half-plane polar ordering; (e)-(h) upper half-plane $\alpha$-polar ordering, with four values of $\alpha$. In all the cases the structuring element $B$ is also a square of $5 \times 5$ pixels. 

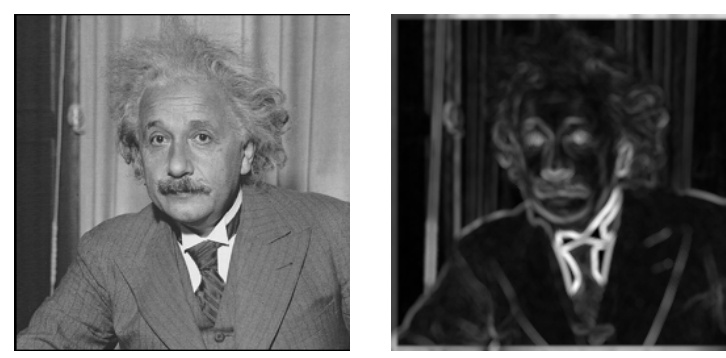

(a) $f(p)$
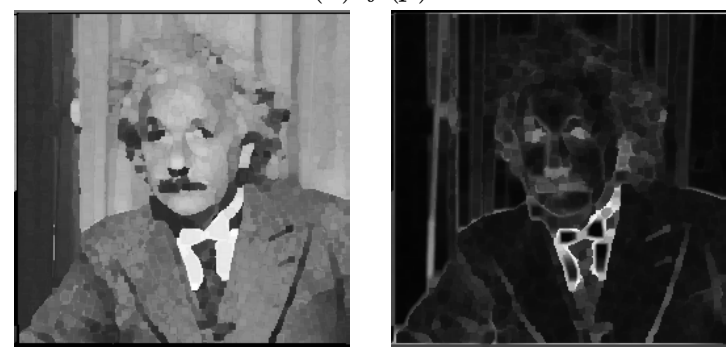

(c) $\delta_{B}(f)(p),\left(\mathcal{H}^{2}, \leq_{\mathcal{H}^{2}}^{\text {polar }}\right)$
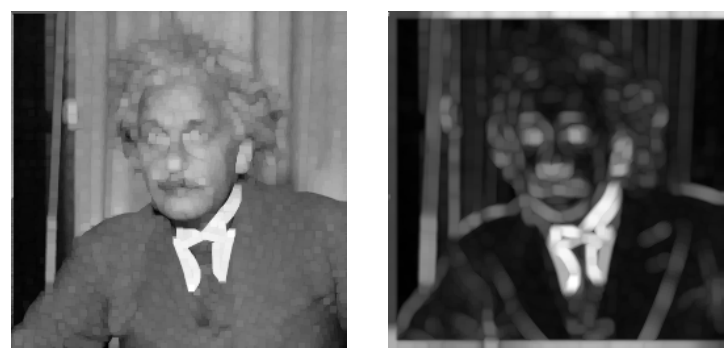

(b) $\delta_{B}(f)(p),\left(\mathcal{H}^{2}, \leq_{\mathcal{H}^{2}}\right)$
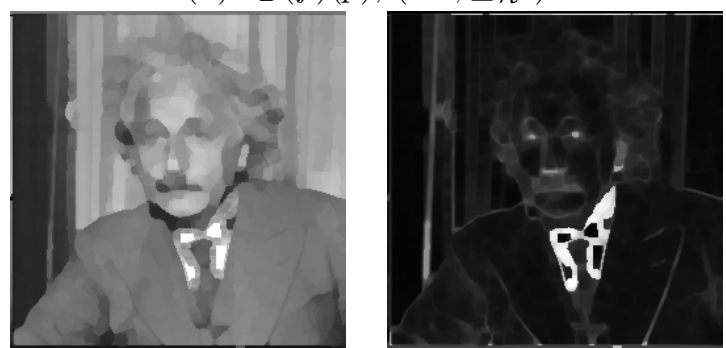

(d) $\delta_{B}(f)(p),\left(\mathcal{H}^{2}, \leq_{\mathcal{H}^{2}}^{\alpha-p o l}\right), \alpha=0.01$

Figure 14: Comparison of dilation of Gaussian distribution-valued image $\delta_{B}(f)(p)$ : (a) Original image $f \in \mathcal{F}\left(\Omega, \mathcal{H}^{2}\right)$, showing both the real and the imaginary components; (b) upper half-plane product ordering (equivalent to standard processing); (c) upper half-plane polar ordering; (e) half-plane $\alpha$-polar ordering, with $\alpha=0.01$. In all the cases the structuring element $B$ is also a square of $5 \times 5$ pixels. 

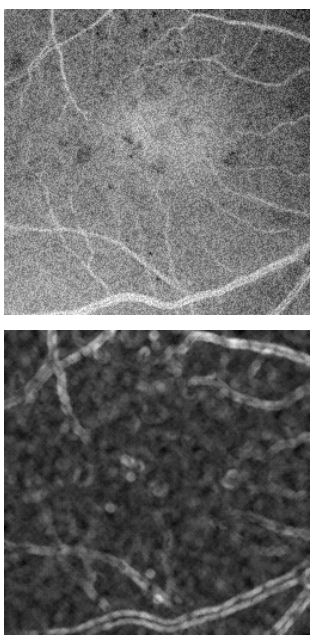

(a) $f(p)$
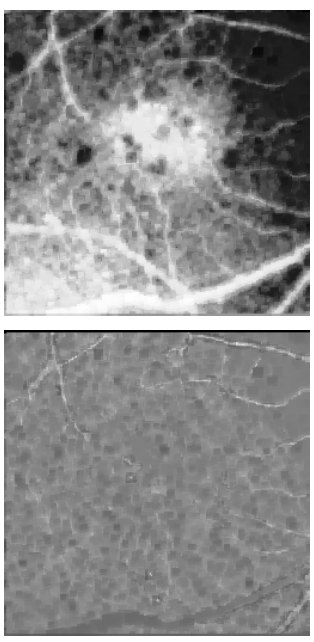

(g) $\gamma_{B}(f)(p)$

$\left(\mathcal{H}^{2}, \bigvee_{\mathcal{H}^{2}}^{g e o}, \bigwedge_{\mathcal{H}^{2}}^{g e o}\right)$
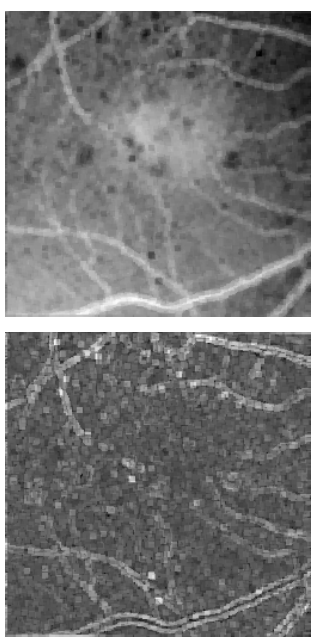

(b) Minimax

center $5 \times 5$
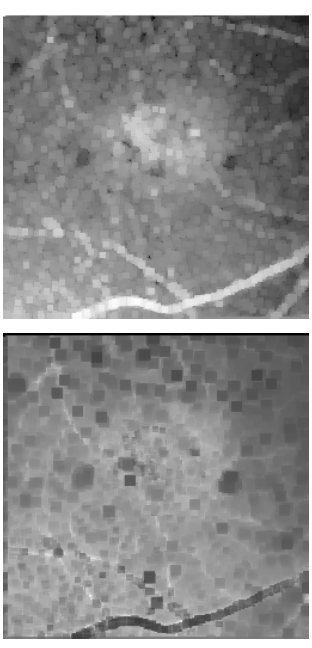

(h) $\gamma_{B}(f)(p)$,

$\left(\mathcal{H}^{2}, \wedge_{\mathcal{H}^{2}}^{-\rightarrow}, \bigvee_{\mathcal{H}^{2}}^{-\rightarrow+}\right)$
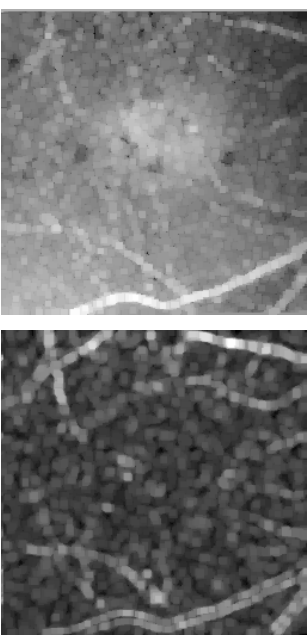

(c) $\gamma_{B}(f)(p)$, $\left(\mathcal{H}^{2}, \leq_{\mathcal{H}^{2}}\right)$
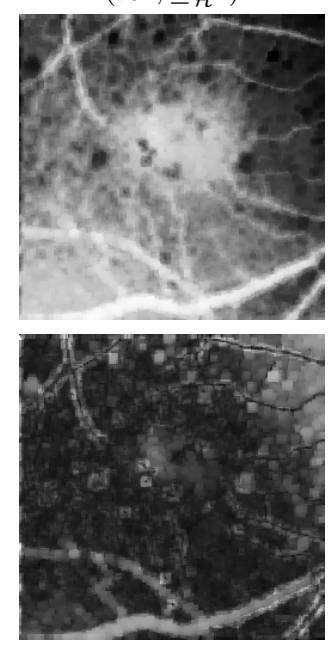

(g) $\varphi_{B}(f)(p)$, $\left(\mathcal{H}^{2}, \bigvee_{\mathcal{H}^{2}}^{g e o}, \bigwedge_{\mathcal{H}^{2}}^{g e o}\right)$
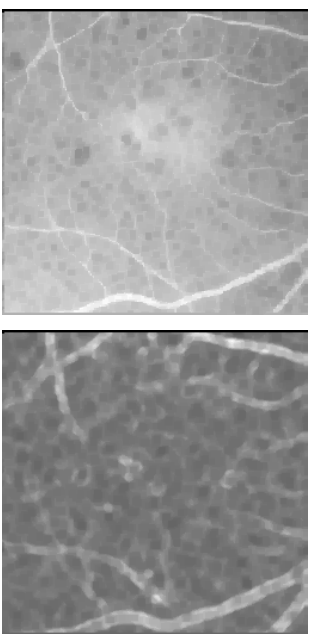

(d) $\varphi_{B}(f)(p)$, $\left(\mathcal{H}^{2}, \leq_{\mathcal{H}^{2}}\right)$
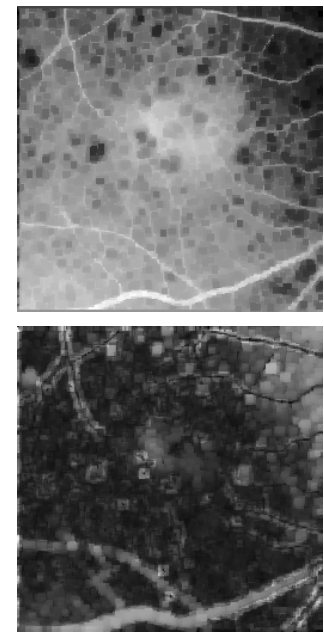

(h) $\varphi_{B}(f)(p)$, $\left(\mathcal{H}^{2}, \bigwedge_{\mathcal{H}^{2}}^{-+}, \bigvee_{\mathcal{H}^{2}}^{-\vec{H}^{+}}\right)$

Figure 15: Morphological processing of Gaussian distribution-valued noisy image: (a) Original image $f \in \mathcal{F}\left(\Omega, \mathcal{H}^{2}\right)$, showing both the real and the imaginary components; (b) filtered image by computing the minimax center in a square of $5 \times 5$ pixels; (c) morphological opening working on the product lattice; (d) morphological closing working on the product lattice; (e) morphological pseudo-opening working on the geodesic lattice; (f) morphological pseudoopening on the asymmetric geodesic lattice; (g) morphological pseudo-closing working on the geodesic lattice; (h) morphological pseudo-closing on the asymmetric geodesic lattice. In all the cases the structuring element $B$ is also a square of $5 \times 5$ pixels. 

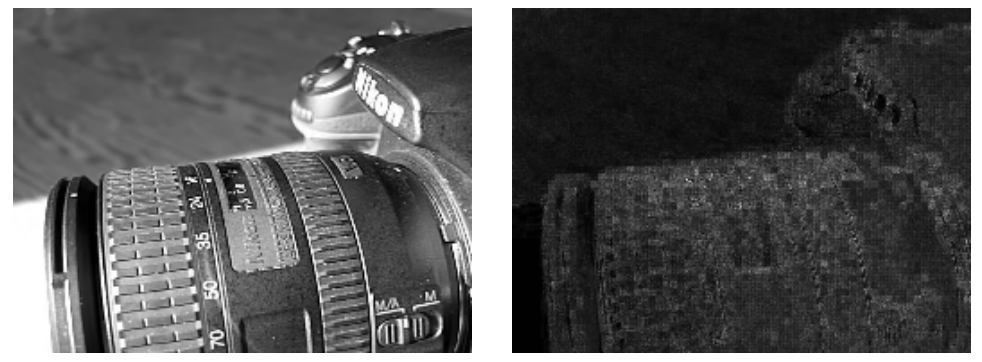

(a) $f(p)$

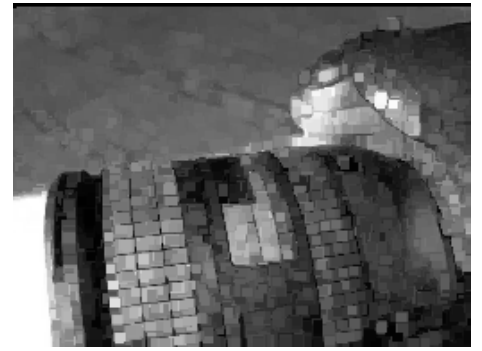

(b) $\hat{f}(p)=\gamma_{B}(f)(p),\left(\mathcal{H}^{2}, \leq_{\mathcal{H}^{2}}^{\text {pol }}\right)$

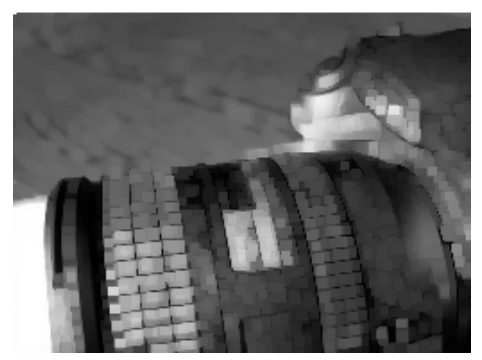

(d) $\check{f}(p)=\gamma_{B}(f)(p),\left(\mathcal{H}^{2}, \bigwedge_{\mathcal{H}^{2}}^{-\rightarrow}, \bigvee_{\mathcal{H}^{2}}^{-\rightarrow+}\right)$
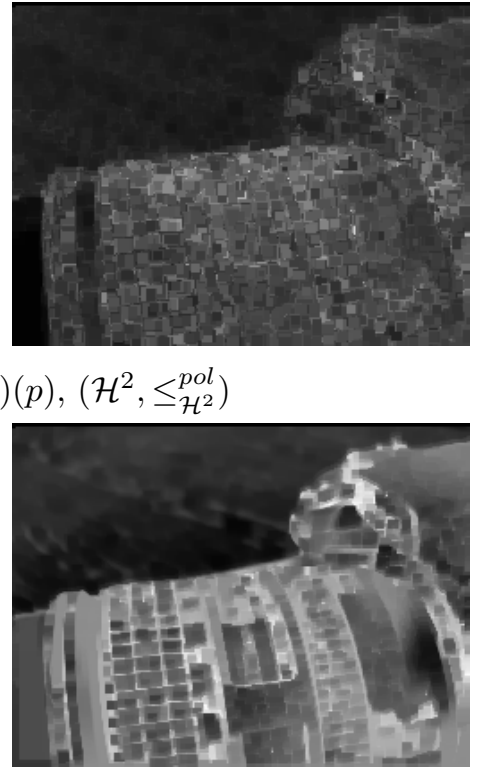

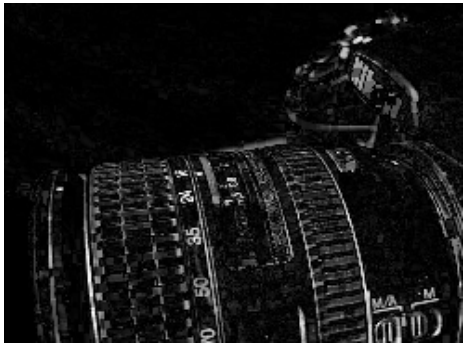

(c) $\operatorname{dist}_{\mathcal{H}^{2}}(f(p), \hat{f}(p))$

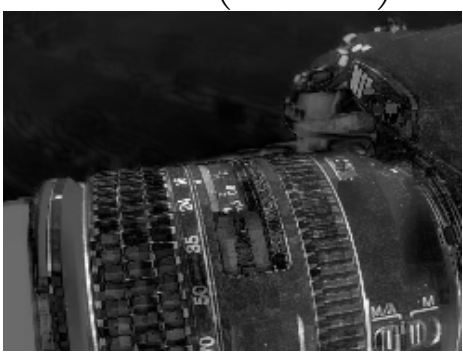

(e) $\operatorname{dist}_{\mathcal{H}^{2}}(f(p), \check{f}(p))$

Figure 16: Morphological detail extraction of multiple acquisition image modeled as a Gaussian distribution-valued: (a) Original image $f \in \mathcal{F}\left(\Omega, \mathcal{H}^{2}\right)$, showing both the real and the imaginary components; (b) morphological opening $\gamma_{B}(f)$ working on polar ordering-based lattice; (c) corresponding residue (pixelwise hyperbolic difference) between the original and the opened image; (d) morphological pseudo-opening $\gamma_{B}(f)$ working on the asymmetric geodesic lattice; (e) corresponding residue. In both cases the structuring element $B$ is also a square of $7 \times 7$ pixels. 


\section{Perspectives}

Levelings are a powerful family self-dual morphological operators which have been also formulated in vector spaces [24], using geometric notions as minimum enclosing balls and half-planes intersection. We intend to explore the formulation of levelings in the upper half-plane in a future work.

The complete lattice structures for the Poincaré upper-half plane introduced in this work, and corresponding morphological operators, can be applied to process other hyperbolic-valued images. For instance, on the one hand, it was proven in [13] that the structure tensor for 2D images, i.e., at each pixel is given a $2 \times 2$ symmetric positive definite matrix which determinant is equal to 1, are isomorphic to the Poincaré unit disk model. On the other hand, polarimetric images [17] where at each pixel is given a partially polarized state can be embedded in the Poincaré unit disk model. In both cases, we only need the mapping from the Poincaré disk model to the Poincaré half-plane, i.e.,

$$
z \mapsto-i \frac{z+1}{z-1}
$$

We have considered here the case of Gaussian distribution-valued images. It should be potentially interesting for practical applications to consider that the distribution of intensity at a given pixel belongs to a more general distributions compared to the Gaussian one. In particular, the case of the Gamma distribution seems an appropriate framework. The information geometry of the gamma manifold has been studied in the past [16] and some of the ideas developed in this work can be revisited for the case of Gamma-distribution valued images by endowing the gamma manifold of complete lattice structure.

Previous extension only concerns the generalization of ordering structure for univariate distributions. In the case of multivariate Gaussian distributions, we can consider to replace the Poincaré upper-half plane by the Siegel upper-half space [7].

\section{References}

[1] J. Angulo, S. Velasco-Forero. Complete Lattice Structure of Poincaré Upper-Half Plane and Mathematical Morphology for Hyperbolic-Valued Images. In Proc. of First International Conference Geometric Science of Information (GSI'2013), (F. Nielsen, F. Barbaresco, Eds.) Springer LNCS Vol. 8085, pp. 535-542, 2013.

[2] M. Arnaudon, F. Nielsen. On approximating the Riemannian 1-center. Computational Geometry, Vol. 46, No. 1, 93-104, 2013.

[3] S.-I. Amari, O.E. Barndorff-Nielsen, R.E. Kass, S.L. Lauritzen, C.R. Rao. Differential Geometry in Statistical Inference. Institute of Mathematical Statistics, Lecture NotesMonograph Series Vol. 10, 1987. 
[4] S.-I. Amari, H. Nagaoka. Methods of Information Geometry, Translations of Mathematical Monographs. Vol. 191, Am. Math. Soc., 2000.

[5] F. Barbaresco. Interactions between Symmetric Cone and Information Geometries: : Bruhat-Tits and Siegel Spaces Models for High Resolution Autoregressive Doppler Imagery. In (F. Nielsen, Eds.) Emerging Trends in Visual Computing (ETCV'08), Springer LNCS Vol. 5416, pp. 124-163, 2009.

[6] F. Barbaresco. Geometric radar processing based on Fréchet distance: information geometry versus optimal transport theory. In Proc. of IEEE International Radar Symposium (IRS'2011), pp. 663-668, 2011.

[7] F. Barbaresco. Information Geometry of Covariance Matrix: Cartan-Siegel Homogeneous Bounded Domains, Mostow/Berger Fibration and Fréchet Median. In (F. Nielsen, $R$. Bhatia, Eds.) Matrix Information Geometry, Springer, pp. 199-255, 2013.

[8] M. Bădoiu, K.L. Clarkson. Smaller core-sets for balls. In Proc. of the fourteenth annual ACM-SIAM symposium on Discrete algorithms (SIAM), pp. 801-802, 2003.

[9] J. Burbea, C.R. Rao. Entropy differential metric, distance and divergence measures in probability spaces: A unified approach. Journal of Multivariate Analysis, Vol. 12, No. 4, 575-596, 1982.

[10] A. Căliman, M. Ivanovici, N. Richard. Probabilistic pseudo-morphology for grayscale and color images. Pattern Recognition 47: 721-735, 2004.

[11] V. Cammarota, E. Orsingher. Travelling Randomly on the Poincaré Half-Plane with a Pythagorean Compass. Journal of Statistical Physics, Vol. 130, No. 3, 455-482, 2008.

[12] J.W. Cannon, W.J. Floyd, R. Kenyon, W.R. Parry. Hyperbolic Geometry. Flavors of Geometry, MSRI Publications, Vol. 31, 1997.

[13] P. Chossat, O. Faugeras. Hyperbolic Planforms in Relation to Visual Edges and Textures Perception. PLoS Computational Biology, Vol. 5, Issue 12, p1, 2009.

[14] S.I.R. Costa, S.A. Santos, J.E. Strapasson. Fisher information matrix and hyperbolic geometry. In Proc. of IEEE ISOC ITW2005 on Coding and Complexity, pp. 34-36, 2005 .

[15] S.I.R. Costa, S.A. Santos, J.E. Strapasson. Fisher information distance: a geometrical reading, arXiv:1210:2354v1, 15 p., 2012.

[16] C.T.J Dodson, H. Matsuzoe. An affine embedding of the gamma manifold. Appl. Sci., $5(1): 7-12,2003$ 
[17] J. Frontera-Pons, J. Angulo. Morphological Operators for Images Valued on the Sphere. In Proc. of IEEE ICIP'12 (2012 IEEE International Conference on Image Processing), pp. 113-116, Orlando (Florida), USA, October 2012.

[18] L. Fuchs. Partially ordered algebraic systems. Pergamon, 1963.

[19] A.K. Guts. Mappings of families of oricycles in Lobachevsky space. Math. USSR-Sb., Vol. 19, 131-138, 1973.

[20] A.K. Guts. Mappings of an ordered Lobachevsky space. Siberian Math. J., Vol. 27, No. 3, 347-361, 1986.

[21] H.J.A.M. Heijmans. Morphological image operators. Academic Press, Boston, 1994.

[22] H.J.A.M. Heijmans, R. Keshet. Inf-semilattice approach to self-dual morphology. Journal of Mathematical Imaging and Vision, Vol. 17, No. 1, 55-80, 2002.

[23] R. Keshet. Mathematical Morphology on Complete Semilattices and its Applications to Image Processing. Fundamenta Informatica, Vol. 41, 33-56, 2000.

[24] F. Meyer. Vectorial Levelings and Flattenings. In Mathematical Morphology and its Applications to Image and Signal Processing (Proc. of ISMM'02), Kluwer, pp. 51-60, 2000.

[25] F. Nielsen, R. Nock. On the smallest enclosing information disk. Information Processing Letters, Vol. 105, 93-97, 2008.

[26] F. Nielsen, R. Nock. Hyperbolic Voronoi diagrams made easy. In Proc. of the 2010 IEEE International Conference on Computational Science and Its Applications, pp. 74-80, 2010.

[27] Z. Sachs. Classification of the Isometries of the upper half-plane. University of Chicago VIGRE REU, 14 p., 2011.

[28] L. Sbaiz, F. Yang, E. Charbon, S. Süsstrunk, M. Vetterli. The Gigavision Camera. In Proc. of IEEE ICASSP'09, pp. 1093-1096, 2009.

[29] J. Serra. Image Analysis and Mathematical Morphology. Vol II: Theoretical Advances, Academic Press, London, 1988.

[30] M. Shaked, J. G. Shanthikumar. Stochastic Orders and their Applications, Associated Press, 1994.

[31] P. Soille. Morphological Image Analysis. Springer-Verlag, Berlin, 1999.

[32] A. Treibergs. The Hyperbolic Plane and its Immersions into $\mathbb{R}^{3}$, Lecture note. Department of Mathematics, University of Utah, 13 p., 2003. 